\title{
From two types of electrostatic position-dependent semiconductor qubits to quantum universal gates and hybrid semiconductor- superconducting quantum computer
}

Krzysztof Pomorski, Panagiotis Giounanlis, Elena Blokhina, Dirk Leipold, Paweł Pęczkowski, et al.

Krzysztof Pomorski, Panagiotis Giounanlis, Elena Blokhina, Dirk Leipold, Paweł Pęczkowski, Robert Bogdan Staszewski, "From two types of electrostatic position-dependent semiconductor qubits to quantum universal gates and hybrid semiconductor-superconducting quantum computer," Proc. SPIE 11054, Superconductivity and Particle Accelerators 2018, 110540M (14 May 2019); doi: 10.1117/12.2525217

EDEent: Superconductivity and Particle Accelerators 2018, 2018, Krakow, Poland 


\title{
From Two Types of Electrostatic Position-Dependent Semiconductor Qubits to Quantum Universal Gates and Hybrid Semiconductor-Superconducting Quantum Computer
}

\author{
Krzysztof Pomorski $^{a}$, Panagiotis Giounanlis ${ }^{a}$, Elena Blokhina ${ }^{a}$, \\ Dirk Leipold ${ }^{b}$, Paweł Pęczkowski ${ }^{c}$, and Robert Bogdan Staszewski ${ }^{a}$ \\ ${ }^{a}$ University College Dublin, School of Electrical and Electronic Engineering, Dublin 4, Ireland; \\ ${ }^{b}$ Equal 1 Company, California, USA; \\ ${ }^{c}$ Cardinal Stefan Wyszyński University, Faculty of Mathematics and Natural Sciences, \\ School of Exact Sciences, Department of Physics, K. Wóycickiego 1/3, 01-938 Warsaw, Poland
}

\begin{abstract}
Properties of two types of position-dependent electrostatic qubits: eigenenergy-based and Wannier-based, are treated with Schrödinger formalism. Their operating principles are given. The corresponding quantum universal gates for selected qubit types are described and their possible implementation is suggested. The modeling methodology of setting and reading semiconductor qubit is suggested. The interface between superconducting and semiconductor quantum computer is proposed and its implementation and operating principles are described.
\end{abstract}

Keywords: Semiconductor qubit, position-dependent qubit, superconductor-semiconductor qubit interface, quantum gates, quantum computing.

\section{INTRODUCTION}

Currently, a heated world-wide race towards construction of a quantum computer is taking place. ${ }^{1-5}$ Although at present the superconducting technologies are dominant, ${ }^{6}$ we claim that CMOS semiconductor technologies appear to have a potential of much better scalability than the superconducting qubits do,$^{7}{ }^{8}$ We also consider the interface between semiconductor and superconducting types of quantum computers that is based on interface between superconducting flux vs. position-dependent semiconductor electrostatic qubit and interface between Cooper pair box and semiconductor position-dependent qubit. So far, the usage of electron is the most common means of transporting and manipulating the information and indeed the proposed position-dependent electrostatic qubits are relying on the use of single electrons being confined by local potentials controlled by CMOS gates. In this work, we describe the classical and quantum computer that can be implemented by using analogy of charged balls that can be scaled down to the size of a single electron. Such an electron can move in semiconductor nanowires. One can use voltage across the gates that are in a proximity to the nanowire to bring an electron from one geometric place into another. At the same time, we can exploit the anticorrelation of electrons' positions that is depicted in Fig. 1(A) and which comes from minimization of electrostatic energy. Briefly, two electrons in two separated boxes take up positions that set their maximum distance due to the presence of Coulomb interaction. Using the concept of three electrons in three boxes, we arrive at the idea of classical and quantum CNOT gate that is given in Fig. 1(B). Desiring to use concepts (A) and (B) in a quantum regime, one needs to refer to the concept of position-dependent qubit as a bit of information distributed between two neighbouring wells, as depicted by Fig. 1(C). All these concepts will be incorporated into Schröedinger formalism.

Using these concepts, we will show preliminary schemes of quantum universal gates. In particular, we exploit electrostatic Coulomb interaction between the trapped electrons and quantum coherence of interacting electrons.

Further author information: (Send correspondence to Krzysztof Pomorski)

A.A.A.: E-mail: kdvpomorski@gmail.com, Telephone: +48 728384555

B.B.A.: E-mail: krzysztof.pomorski@ucd.ie

Superconductivity and Particle Accelerators 2018, edited by Dariusz Bocian, Ryszard S. Romaniuk,

Proc. of SPIE Vol. 11054,110540M · (c) The Authors. Published under a Creative Commons

Attribution CC-BY 3.0 License · doi: 10.1117/12.2512039

Proc. of SPIE Vol. 11054 110540M-1 

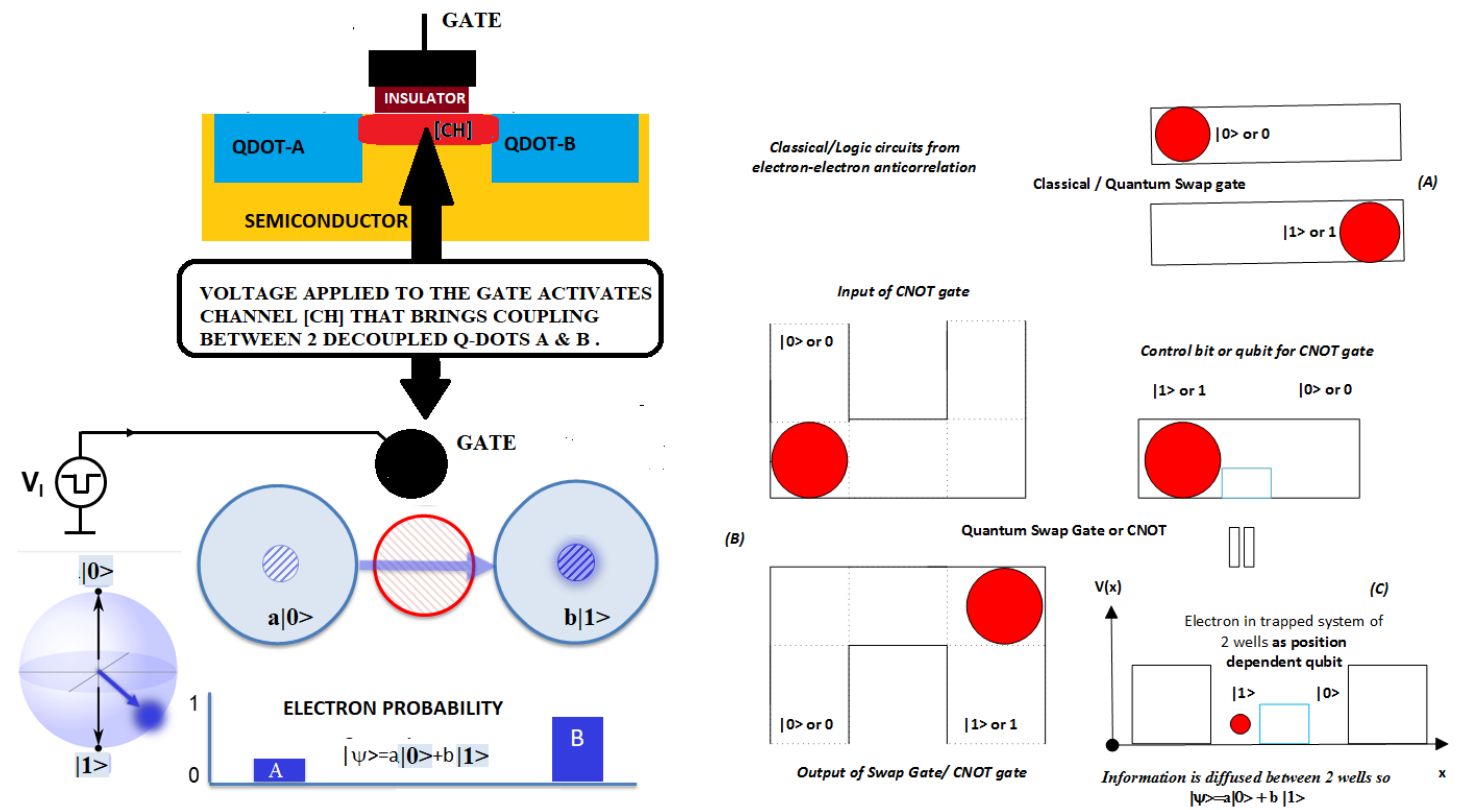

Figure 1. Technological definition of electrostatic position-based qubit and related three basic concepts: (A) anticorrelation of positions due to Coulomb force, (B) classical and quantum CNOT gate, (C) information based on position.

Essentially, we exploit the approximation of an electron in an effective potential and readjust numerical solutions for the case of weak perturbative 2- and $N$-body interactions. Such 2-body interaction brings dephasing of the two systems. At a bigger scale, it is known in solid-state physics that quantum states shall be described by Random Phase Approximation.

With a proper choice of implementation scheme, it is possible to limit the description of physical processes to a quasi one-dimensional (1D) case. Many phenomena taking place in two and three dimensions can be effectively described with $1 \mathrm{D}$ models, which significantly reduce their numerical computation and analysis complexity. In such a way, for example, instead of dealing with partial differential equations, we can use ordinary differential equations. Once the computation is performed in 1D, it is then possible to extend it to higher dimensions. Such approach allows to understand the obtained results in a better way. On the other hand, 1D systems are known to interact much stronger than the $2 \mathrm{D} / 3 \mathrm{D}$ systems do. Hence, there is a methodological motivation to start from 1D models.

A qubit is traditionally defined as a quantum system with two discrete energy levels, whose occupancy can be regulated in a technologically specific manner, e.g. via the use of electric or magnetic fields. In case of a quantum dot, the regulating factor is usually an electric field that "pushes" away or injects an electron into a quantum dot. Spectral theorem guarantees that two different eigenvalues of the Hamiltonian $H$ will have orthogonal eigenstates. This is an essential property for the qubit operation. In our case, it is necessary to have at least two energy levels in the system. The occupancy of the left well is given as $\int_{w_{L}}|\psi|^{2} d x=|a|^{2}$ and denoted in the qubit abstract representation by $|a|$, while the occupancy of the neighbouring right well is given by $\int_{w_{R}}|\psi|^{2} d x=|b|^{2}$ and denoted by $|b|$. Obviously, $|a|^{2}+|b|^{2}=1$. The given reasoning shall be valid for one electron confined in two wells but also for $N$ electrons confined within two wells. In case of the $N$-electron presence, we shall deal with effective phenomenological macroscopic wavefunction. At this stage, a reference to the mean field theory is also useful. We denote the functional dependence with '[]' brackets, the Hamiltonian as $H$ and geometrical details by $G$. In such a way, the qubit state can be formally written as

$$
|\psi\rangle=a[\psi(x)]|0[\psi(x)], H, G\rangle+b[\psi(x)]|1[\psi(x)], H, G\rangle=e^{i \gamma}\left(F_{L}[\psi(t)]|0\rangle\right)+\left(F_{R}[\psi(t)]|1\rangle\right) .
$$


We notice that $F_{L}[\psi(t)]$ and $F_{R}[\psi(t)]$ are functionals of wavefunctions (as for example integrals of electron density from $-\infty$ to the middle point of barrier separating two wells in position dependent qubit from Fig. 1 as it is the case of $F_{L}$ ) and their general properties are quite peculiar. In a most exotic representation, $|0[\psi]\rangle$ or $|1[\psi]\rangle$ can have a polynomial dependence on Hamiltonian eigenfunctions $\psi_{0}(x), \psi_{0}(1)$. The functional dependence of coefficients $a$ and $b$ on the wavefunction can reflect the way the measurement apparatus reacts to the dynamic state of quantum system. An obvious candidate for qubit representation is a linear combination of two energy levels for 'logic' $|0\rangle$ and linear combination of energy level for 'logic' $|1\rangle$.

Now we are approaching methodology of positon based electrostatic qubit that was firstly reported by ${ }^{9}$ and. ${ }^{10}$ We represent the quantum state as a vector of two eigenfunctions with neglected spin. We consider two energy levels with probability of occupancy $\left|q_{0}\right|^{2}$ and $\left|q_{1}\right|^{2}$, such that $\left|q_{0}\right|^{2}+\left|q_{1}\right|^{2}=1$. We write the quantum state in the form

$$
|\psi(x, t)\rangle=\left[\begin{array}{ll}
1 & 1
\end{array}\right]\left[\begin{array}{cc}
q_{0}(t) & 0 \\
0 & q_{1}(t)
\end{array}\right]\left[\begin{array}{l}
\psi_{0}(x) \\
\psi_{1}(x)
\end{array}\right] .
$$

Now, we introduce the unitary matrix $U$ that fulfills the condition $\hat{U} \hat{U}^{\dagger}=I$. Thus, we obtain

$$
|\psi(x, t)\rangle=\left[\begin{array}{ll}
1 & 1
\end{array}\right]\left[\begin{array}{cc}
q_{0}(t) & 0 \\
0 & q_{1}(t)
\end{array}\right] \hat{U} \hat{U}^{\dagger}\left[\begin{array}{l}
\psi_{0}(x) \\
\psi_{1}(x)
\end{array}\right] .
$$

Then, we specify unitary matrix structure to be

$$
\hat{U}=\left[\begin{array}{cc}
c_{0} & c_{1} \\
c_{1}^{\dagger} & c_{2}
\end{array}\right], \quad \hat{U}^{\dagger}=\left[\begin{array}{cc}
c_{0}^{\dagger} & c_{1}^{\dagger} \\
c_{1} & c_{2}^{\dagger}
\end{array}\right]
$$

We impose unitarian condition that is

$$
\hat{U} \hat{U}^{\dagger}=\left[\begin{array}{cc}
c_{0} & c_{1} \\
c_{1}^{\dagger} & c_{2}
\end{array}\right]\left[\begin{array}{cc}
c_{0}^{\dagger} & c_{1}^{\dagger} \\
c_{1} & c_{2}^{\dagger}
\end{array}\right]=\left[\begin{array}{cc}
\left|c_{0}\right|^{2}+c_{1}^{2} & c_{0} c_{1}^{\dagger}+c_{1} c_{2}^{\dagger} \\
c_{0}^{\dagger} c_{1}^{\dagger}+c_{1} c_{2} & \left|c_{2}\right|^{2}+\left(c_{1}^{\dagger}\right)^{2}
\end{array}\right]=\left[\begin{array}{cc}
1 & 0 \\
0 & 1
\end{array}\right] .
$$

We notice that $c_{1}$ has only real values since the elements on the diagonal on the right-hand side of $U U^{\dagger}$ are real valued. Therefore $c_{1}= \pm c_{1}^{\dagger}$ with a consequence that $\left|c_{0}\right|=\left|c_{2}\right|$. Setting

$$
\hat{U}=\exp (i \alpha)\left[\begin{array}{cc}
i\left|c_{0}\right| & -\left|c_{1}\right| \\
\left|c_{1}\right| & -i\left|c_{0}\right|
\end{array}\right]
$$

and with assumption $\left|c_{0}\right|^{2}+\left|c_{1}\right|^{2}=1$ (since $\operatorname{det}(U)=1$ ) we have fulfilled the unitarity condition $\hat{U} \hat{U}^{\dagger}=I$.

We now define a new orthogonal base for a Wannier position-dependent qubit. We have

$$
\left[\begin{array}{l}
w_{0}(x) \\
w_{1}(x)
\end{array}\right]=\left[\begin{array}{cc}
-i\left|c_{0}\right| & \left|c_{1}\right| \\
-\left|c_{1}\right| & i\left|c_{0}\right|
\end{array}\right]\left[\begin{array}{l}
\psi_{0}(x) \\
\psi_{1}(x)
\end{array}\right]=\left[\begin{array}{l}
-i\left|c_{0}\right| \psi_{0}(x)+\left|c_{1}\right| \psi_{1}(x) \\
-\left|c_{1}\right| \psi_{0}(x)+i\left|c_{0}\right| \psi_{1}(x)
\end{array}\right] .
$$

The quantities $w_{0}(x)$ and $w_{1}(x)$ turn out to be Wannier functions. They are maximally localized at each well with a proper choice of $c_{0}$. Finally, we have the wavefunction expressed by the linear combination of Wannier functions as

$$
\begin{aligned}
& |\psi(x, t)\rangle=\left[\begin{array}{ll}
1 & 1
\end{array}\right]\left[\begin{array}{cc}
q_{0}(t) & 0 \\
0 & q_{1}(t)
\end{array}\right]\left[\begin{array}{cc}
+i\left|c_{0}\right| & -\left|c_{1}\right| \\
\left|c_{1}\right| & -i\left|c_{0}\right|
\end{array}\right]\left[\begin{array}{l}
-i\left|c_{0}\right| \psi_{0}(x)+\left|c_{1}\right| \psi_{1}(x) \\
-\left|c_{1}\right| \psi_{0}(x)+i\left|c_{0}\right| \psi_{1}(x)
\end{array}\right]=
\end{aligned}
$$

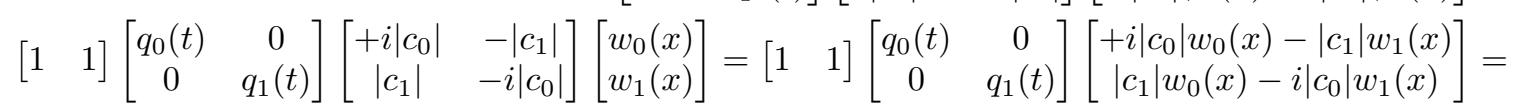

$$
\begin{aligned}
& {\left[\begin{array}{ll}
1 & 1
\end{array}\right]\left[\begin{array}{c}
q_{0}(t)\left(+i\left|c_{0}\right| w_{0}(x)-\left|c_{1}\right| w_{1}(x)\right) \\
q_{1}(t)\left(\left|c_{1}\right| w_{0}(x)-i\left|c_{0}\right| w_{1}(x)\right)
\end{array}\right]} \\
& =q_{0}(t)\left(+i\left|c_{0}\right| w_{0}(x)-\left|c_{1}\right| w_{1}(x)\right)+q_{1}(t)\left(\left|c_{1}\right| w_{0}(x)-i\left|c_{0}\right| w_{1}(x)\right)= \\
& w_{0}(x)\left(q_{0}(t) i\left|c_{0}\right|+q_{1}(t) \sqrt{1-\left|c_{0}\right|^{2}}\right)+w_{1}(x)\left(q_{1}(t)-i\left|c_{0}\right|-q_{0}(t)\left|c_{1}\right|\right)=|\psi(x, t)\rangle .
\end{aligned}
$$


It turns out that the most effective description of the poly-energetic electrostatic semiconductor qubit is by Wannier functions, ${ }^{11}$ while the most effective description of the mono-energetic electrostatic semicondcutor qubit, as later depicted in Fig. 5, is by Hamiltonian eigenfunctions (wavefunctions). The second type of the position-dependent qubit will be described later.

The issue of projection and measurement in the position-dependent qubit can be seen in the following short reasoning. Now we are concentrating on the first type of position-dependent qubit that requires the usage of Wannier functions in order to represent logic $|0\rangle$ and $|1\rangle$. By measuring a particle in $w_{L}$ (left well) we make the use of the projection operator

$$
\int_{x 1}^{x 2}|x\rangle\langle x| d x
$$

on a given quantum state $|\psi\rangle$. Since the given quantum state for the two-level system has the form $|\psi\rangle=$ $q_{0}|E\rangle_{0}+q_{1}|E\rangle_{1}$ we can write the projection operator acting on $|\psi\rangle$ as

$$
\begin{array}{r}
\hat{P}_{\left(x_{1}, x_{2}\right) \in w_{L}}=\left(\int_{x 1}^{x 2}|x\rangle\langle x| d x\right)|\psi\rangle= \\
=\int_{x 1}^{x 2} \psi(x)|x\rangle=\left(\int_{x 1}^{x 2}|x\rangle\langle x| d x\right)\left(q_{0}|E\rangle_{0}+q_{1}|E\rangle_{1}\right)= \\
=q_{0}\left(\int_{x 1}^{x 2} \psi(x)_{0}|x\rangle d x\right)+q_{1}\left(\int_{x 1}^{x 2} \psi(x)_{1}|x\rangle d x\right) .
\end{array}
$$

Clearly, we have a linear combination of two energy projections in the act of measurement done on the left well. We underline that the final expression is technically challenging as it must be limited only to the action on the left or right well. Shining light on the left well might bring the zero ground state $E_{0}$ into $E_{2}$ if the quantum of light has energy $E_{2}-E_{0}$ with probability proportional to $q_{0}$. At the same time, shining light on the left well of energy $E_{2}-E_{1}$ we bring $E_{1}$ into $E_{2}$ with probability $q_{1}$. This processes affects the total quantum state by the measurement done on the left well. Alternatively, for a measurement done via energy projection, we could exploit Rabi oscillations.

Basically, we can use the left well for generating the Hamiltonian part

$$
H_{1_{L}}(t)=f_{L}(t) g_{L}(x)\left[\left|E_{0}\right\rangle\left\langle E_{1}|+| E_{1}\right\rangle\left\langle E_{0}\right|\right] .
$$

This Hamiltonian promotes the eigenstate that is a linear combination of $\left|E_{0}\right\rangle$ and $\left|E_{1}\right\rangle$. It is the best if we apply

$$
f_{L}(t)=a_{0_{L}} \sin \left(t\left(E_{1}-E_{0}\right) / \hbar\right)+b_{0_{L}} \cos \left(t\left(E_{1}-E_{0}\right) / \hbar\right)
$$

so $g_{L}(x)$ can vanish in the right well (or, more precisely, it will have an exponentially decaying tail in the right well).

Action of an AC polarizing voltage of the left gate will regulate occupancy of the left well. In such a way, for activating the left voltage gate for certain time, we can control quantum state in the left well. Therefore, the left gate shall be used for setting the quantum state of left well.

At the same time, we can use the right electrode to extend its action on the right well. Basically we can use the right well for generating the Hamiltonian part

$$
H_{1_{R}}(t)=f_{R}(t) g_{R}(x)\left[|E\rangle_{0}\left\langle\left. E\right|_{1}+\mid E\right\rangle_{1}\left\langle\left. E\right|_{0}\right] .\right.
$$

It is the best if we apply

$$
f_{R}(t)=a_{0_{R}} \sin \left(t\left(E_{1}-E_{0}\right) / \hbar\right)+b_{0_{R}} \cos \left(t\left(E_{1}-E_{0}\right) / \hbar\right)
$$

so $g_{R}(x)$ will vanish in the left well (or, more precisely, will have an exponentially decaying tail there). Furthermore, the right gate shall be used for setting the state in the right well. 
Top view of readout circuit of position dependent qubit

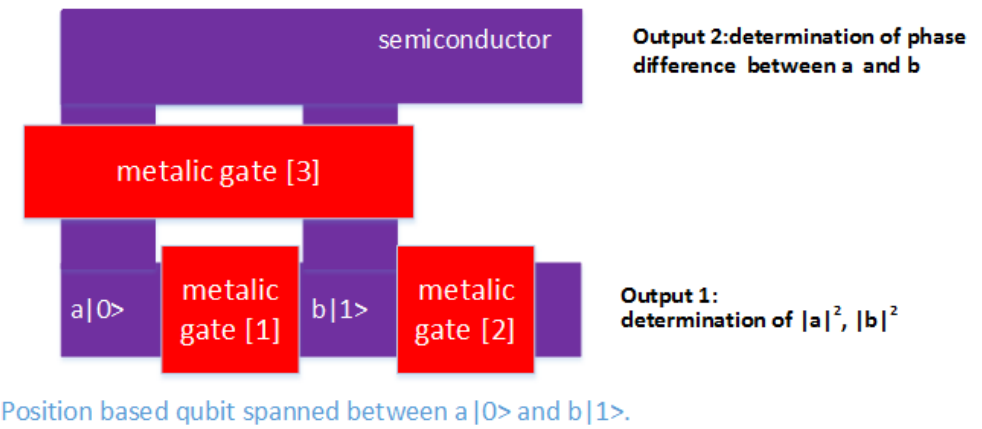

Figure 2. Qubit readout circuit for two types of electrostatic position-dependent semiconductor qubits whose topology is shown in upper part of Fig. 1.

\subsection{Reading the Magnitudes of $a$ and $b$ and their Phase Difference in Two Types of Position-Based Qubits}

The first type of electrostatic position-based semiconductor qubit is a "Wannier qubit". It is illustrated in Fig. 2 and was introduced in. ${ }^{11}$ Essentially, the quantum state is confined by two voltage potentials $V_{\text {out } 2}(t)$ and $V_{\text {out } 3}(t)$ and a barrier separating $a|0\rangle$ from $b|1\rangle$, which is controlled by $V_{\text {out } 1}(t)$. Lifting the barrier controlled by $V_{\text {out } 3}(t)$ will set forth the propagation of wavepackets from the geometrically defined areas where the presence of $a|0\rangle$ and $b|1\rangle$ is prominent. These wavepackets will propagate around the defined paths and a certain level of interference will take place, as it defines the measure of phase shift between $a$ and $b$ coefficients. On the other hand, raising the barrier controlled by $V_{\text {out } 2}(t)$ and, at the same time, the barrier controlled by $V_{\text {out } 1}(t)$, will bring the wavepackets coming from the $a|0\rangle$ and $b|1\rangle$ qubit area. Proper measurement of wavepackets by a single electron transistor (SET1) device will allow the detection of $|a|$ and $|b|$ magnitudes. This area of detection is given by Readout 1 . Also, proper measurement of wavepackets by SET 2 device will allow for the detection of phase difference between $a$ and $b$. In such a way, the basic measurements of two qubit angles on a Bloch sphere can be established.

\subsection{Setting Position-Qubit Phase Difference between $a$ and $b$ by Means of Magnetic and Electric Aharonov-Bohm Effect}

We now describe a situation of a particle under the influence of external magnetic field what is equivalent to the presence of non-zero vector potential in the Schrödinger equation. We start from the situation of one electron in effective potential that is given by the equation

$$
H=-\frac{\hbar^{2}}{2 m_{1}} \frac{d^{2}}{d x^{2}}+V(x)=\frac{1}{2 m_{1}} p_{x}^{2}+V(x) .
$$

In a 1D case with one particle subjected to an external source of vector potential $A_{x}(x)$ as an external polarizing current, we modify the canonical momentum of electron $\hat{p}_{0}=\frac{\hbar}{i} \frac{d}{d x}$ into $\hat{p}_{1}=\frac{\hbar}{i} \frac{d}{d x}-\frac{e}{c} A_{x}(x)$. It brings the following kinetic operator as

$$
\frac{1}{2 m}\left(\frac{\hbar}{i} \frac{d}{d x}-\frac{e}{c} A_{x}(x)\right)^{2}=\frac{1}{2 m}\left(-\hbar^{2} \frac{d^{2}}{d x^{2}}-i \frac{e \hbar}{c} A_{x}(x) \frac{d}{d x}+\left[-i \frac{e \hbar}{c}\left(\frac{d}{d x} A_{x}(x)\right)+\frac{e^{2}}{c^{2}} A_{x}(x)^{2}\right]\right) .
$$

We immediately recognize that

$$
\frac{1}{2 m}\left(\frac{\hbar}{i} \frac{d}{d x}-\frac{e}{c} A_{x}(x)\right)^{2}=\frac{1}{2 m}\left(\hat{p}_{0}^{2}-\frac{e}{c} A_{x}(x) \hat{p}_{0}+\left[-i \frac{e \hbar}{c}\left(\frac{d}{d x} A_{x}(x)\right)+\frac{e^{2}}{c^{2}} A_{x}(x)^{2}\right]\right) .
$$




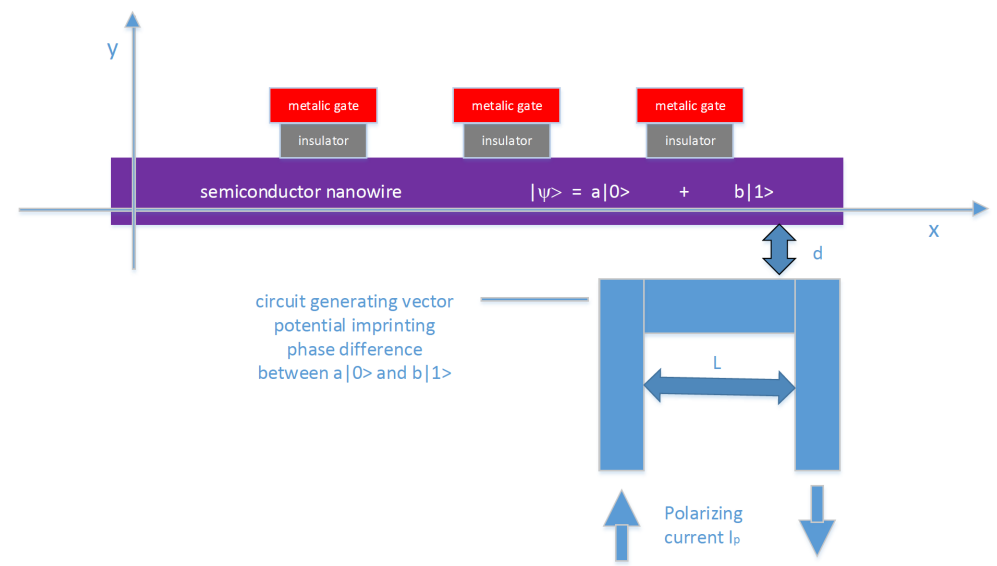

Figure 3. Schematic description of the situation of phase imprint on position dependent qubit where external current source $I_{p}$ is used to generate phase on region of $b|1\rangle$ of position based qubit. It is the example of usage of magnetic AharonovBohm effect for phase control across qubit localized at line $(\mathrm{x}, \mathrm{y}=0)$, so the states $a|0\rangle$ and $b|1\rangle$ exist in neighbouring wells. The alternative phase control across qubit is by the usage of electric Aharonov-Bohm effect as visualized in Fig. 4.

Here, expressions $\frac{e}{c} A_{x}(x) \hat{p}_{0}$ and $-i \frac{e \hbar}{c}\left(\frac{d}{d x} A_{x}(x)\right)$ can be treated as damping or exciting terms. Final Hamiltonian will be modified as

$$
\begin{aligned}
H= & \frac{1}{2 m}\left(\frac{\hbar}{i} \frac{d}{d x}-\frac{e}{c} A_{x}(x)\right)^{2}+V(x)=\frac{1}{2 m}\left(\hat{p}_{0}^{2}-\frac{e}{c} A_{x}(x) \hat{p}_{0}+\left[-i \frac{e \hbar}{c}\left(\frac{d}{d x} A_{x}(x)\right)+\frac{e^{2}}{c^{2}} A_{x}(x)^{2}\right]\right)+V(x)= \\
& =\frac{1}{2 m}\left(\hat{p}_{0}^{2}-\frac{e}{c} A_{x}(x) \hat{p}_{0}+\frac{1}{2 m}\left(\left[-i \frac{e \hbar}{c}\left(\frac{d}{d x} A_{x}(x)\right)+\frac{e^{2}}{c^{2}} A_{x}(x)^{2}\right]\right)+V(x)=\frac{1}{2 m}\left(\hat{p}_{0}^{2}-\frac{e}{c} A_{x}(x) \hat{p}_{0}\right)+V_{1}(x) .\right.
\end{aligned}
$$

We identify the new effective potential $V_{1}(x)$ as coming from the previous potential $\mathrm{V}(\mathrm{x})$ and from a non-zero vector potential. It is given as $V_{1}(x)=\frac{1}{2 m}\left(-i \frac{e \hbar}{c}\left(\frac{d}{d x} A_{x}(x)\right)+\frac{e^{2}}{c^{2}} A_{x}(x)^{2}\right)+V(x)$. We recognize that there appears an imaginary part in the effective $V_{1}$ potential that is due to the presence of the non-zero vector potential. It will cause damping or excitement of the quantum state. From classical mechanics, we know that if the sign of friction coefficient changes, then there will be a transition from damping to excitement (delivery of energy) of a given state. We now consider the situation as depicted in Fig. 3. Vector potential distribution generated by the current source is given by formula $A_{x(y)}(x, y)=\frac{\mu_{0}}{4 \pi} \int d \vec{x}_{1} \cdot \frac{\overrightarrow{j_{x(y)}}\left(\vec{x}_{1}\right)}{\left|\vec{x}-x_{1}\right|}$. In our case with a constant polarizing current $I_{p}$, we have the vector potential in the area of position-dependent qubit

$$
\begin{array}{r}
A_{x}(x, 0)=\frac{\mu_{0} I_{p}}{4 \pi} \int_{0}^{L} \frac{d x_{1}}{\sqrt{\left(x-x_{1}\right)^{2}+d^{2}}=\frac{\mu_{0} I_{p}}{8 \pi}}\left[\log \left(\frac{\sqrt{d^{2}+(x-L)^{2}}-(x-L)}{\sqrt{d^{2}+(x-L)^{2}}+(x-L)}\right)-\log \left(\frac{\sqrt{d^{2}+x^{2}}-x}{\sqrt{d^{2}+x^{2}}+x}\right)\right]= \\
=\frac{\mu_{0} I_{p}}{8 \pi} \log \left[\frac{\left(\sqrt{d^{2}+(x-L)^{2}}-(x-L)\right)\left(\sqrt{d^{2}+x^{2}}+x\right)}{\left(\sqrt{d^{2}+(x-L)^{2}}+(x-L)\right)\left(\sqrt{d^{2}+x^{2}}-x\right)}\right]= \\
=\frac{\mu_{0} I_{p}}{4 \pi}\left[\operatorname{ArcTanh}\left(\frac{L-x}{\sqrt{d^{2}+(x-L)^{2}}}\right)-\operatorname{ArcTanh}\left(\frac{-x}{\sqrt{d^{2}+x^{2}}}\right)\right] .
\end{array}
$$

Therefore, phase difference imprinted on semiconductor qubit line from $(0,0)$ to $(0, L)$ is proportional to

$$
\int_{0}^{L} A_{x}(x, 0) d x=\frac{\mu_{0} I_{p}}{4 \pi} \int_{0}^{L}\left[\operatorname{ArcTanh}\left(\frac{L-x}{\sqrt{d^{2}+(x-L)^{2}}}\right)-\operatorname{ArcTanh}\left(\frac{-x}{\sqrt{d^{2}+x^{2}}}\right)\right] d x .
$$




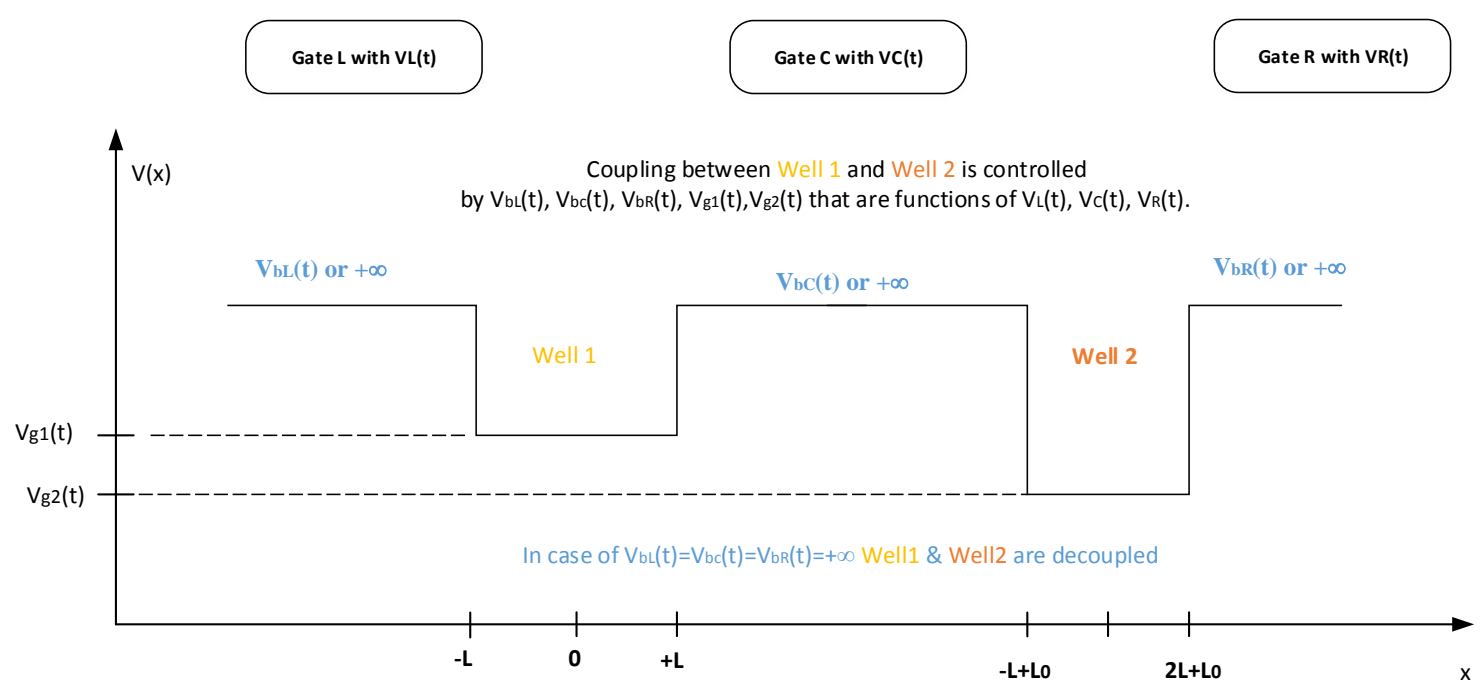

Figure 4. Schematic description of controlling phase difference between two decoupled or partly coupled symmetric wells. It is instructive to refer to Figs. 1 and 5.

Consequently vector potential of biasing current $I_{p}$ generates phase difference across semiconductor nanowire and phase imprint across path from $(0,0)$ to $(\mathrm{L}, 0)$ is given by

$$
\begin{aligned}
& \int_{0}^{L} A_{x}(x, y=0) d x=\frac{\mu I_{p}}{4 \pi}\left(L \log \left(\sqrt{d^{2}+(L-x)^{2}}-(L-x)\right)+\left(\sqrt{d^{2}+(L-x)^{2}}-\sqrt{d^{2}+x^{2}}\right)+\right. \\
&\left.+x\left(\operatorname{ArcTanh}\left(\frac{L-x}{\sqrt{d^{2}+(L-x)^{2}}}\right)+\operatorname{ArcTanh}\left(\frac{x}{\sqrt{d^{2}+x^{2}}}\right)\right)\right)=\alpha(L, y=0) .
\end{aligned}
$$

Therefore, we have the wavefunction before the introduction of vector potential $\psi(x)$ that transfers

$$
\psi(x) \exp \left(i \int_{0}^{L} \frac{e}{\hbar} A_{x}(x, 0) d x\right)=\psi(x) \exp \left(i \frac{e}{\hbar} \alpha(L, 0)\right) .
$$

It is helpful to refer to the physical scenario depicted in Fig. 4 where the two areas of qubit are separated by infinite barriers such that they can be maximally decoupled. Thus, we consider the state localized in the infinite well of length $2 L$ with a flat potential at the bottom $V_{g}$. We can write the effective potential $V(x)$ as follows

$$
V(x)= \begin{cases}V_{g}, & \text { if }-L<x<L . \\ +\infty, & \text { otherwise. }\end{cases}
$$

At the first stage of consideration, $V_{g}$ is time independent, so the lowest energetically allowed wavefunction is of the form $\psi(x)=A \cos \left(\frac{\pi}{2 L} x\right) e^{i \alpha_{0}}$, where $\alpha_{0}$ and $\mathrm{A}$ are constants. From the Schrödigner equation without the presence of vector potential, we obtain the energy $E=\frac{\hbar^{2}}{2 m}\left(\frac{\pi}{2 L}\right)^{2}+V_{g}$. It is the lowest allowed energy of quantum 
state sitting between two infinite potentials that is forced to be time dependent if potential bottom $V_{g}(t)$ is also time-dependent.

It is quite straightforward to obtain the evolution of quantum state of particle confined in rectangular potential with infinite barrier heights and formulas for quantum state evolution with time is $e^{\frac{-i}{\hbar} \int_{t_{0}}^{t} H\left(t^{\prime}\right) d t^{\prime}}=$ $e^{\frac{-i}{\hbar}\left(\left(t-t_{0}\right) \frac{\hbar^{2}}{2 m}\left(\frac{\pi}{2 L}\right)^{2}\right.}+e^{\left.\frac{-i}{\hbar} \int_{t_{0}}^{t} V_{g}\left(t^{\prime}\right)\right) d t^{\prime}}=U(t)=U\left(t, t_{0}\right)$. We recognize that, for the given case, the phase 'imprint' is position independent and is constant for the entire $x$ interval $x \in(-L, L)$. We also notice that in the case of two symmetric wells, we have the time-dependent phase difference given as $\left.\frac{-1}{\hbar} \int_{t_{0}}^{t}\left(V_{g 1}\left(t^{\prime}\right)-V_{g 2}\left(t^{\prime}\right)\right)\right) d t^{\prime}$. In reality, one particle cannot be fully confined within one or two wells of infinite barriers since this would not be achievable from technological point of view where only finite barriers are possible. Thus, a more realistic potential has the structure given in the following way:

$$
V(x, t)= \begin{cases}V_{B}, & \text { if }-L<x \\ V_{g 1}(t), & \text { if }-L<x<L . \\ V_{B}, & \text { if } L<x<L+L 0 \\ V_{g 2}(t), & \text { if } L+L_{0}<x<2 L+L_{0} . \\ V_{B}, & x>2 L+L_{0} .\end{cases}
$$

Basically, we set $V_{B}$ to be large but it is always finite.

\subsection{From Wannier-Based to Eigenenergy-Based Electrostatic Position Semiconductor Qubit}

It is possible to redefine the architecture of position-dependent semiconductor qubit merely by changing the potential bottoms of two neighboring wells. This process is reversible and merely by changing the polarization of three CMOS gates associated with the qubit, we can transform the Wannier qubit into the energy-eigenstate qubit. The situation is depicted in Figs. 5 and 4.

A quantum computer could have three types of quantum universal gates, that is phase rotating gate, Hadamard gate, CNOT gate. In the design of quantum universal gates we need to take into account the following physical factors:

- electron-electron interaction,

- presence of a spin singlet and triplet phase among two and more interacting electrons (so we deal either with antisymmetric or symmetric wavefunction),

- imprint of phase between $a$ and $b$ during the operation of three basic universal gates.

It is possible to obtain the following operations with the use of the above defined quantum state $|\psi\rangle=a|0\rangle+b|1\rangle$

- Imprint of phase on $|\psi\rangle$ due to the magnetic and electric Aharonov-Bohm effect,

- Interference effects allowing the detection of phase difference between $a$ and $b$ coefficients by using different 'optical' paths,

- Decoherence due to tunneling and various enviromental disturbances.

The structure of proposed quantum CNOT and Hadamard gates is described in Fig. 6. Structure of a phase rotating gate is the same as the structure of the position-dependent qubit. 


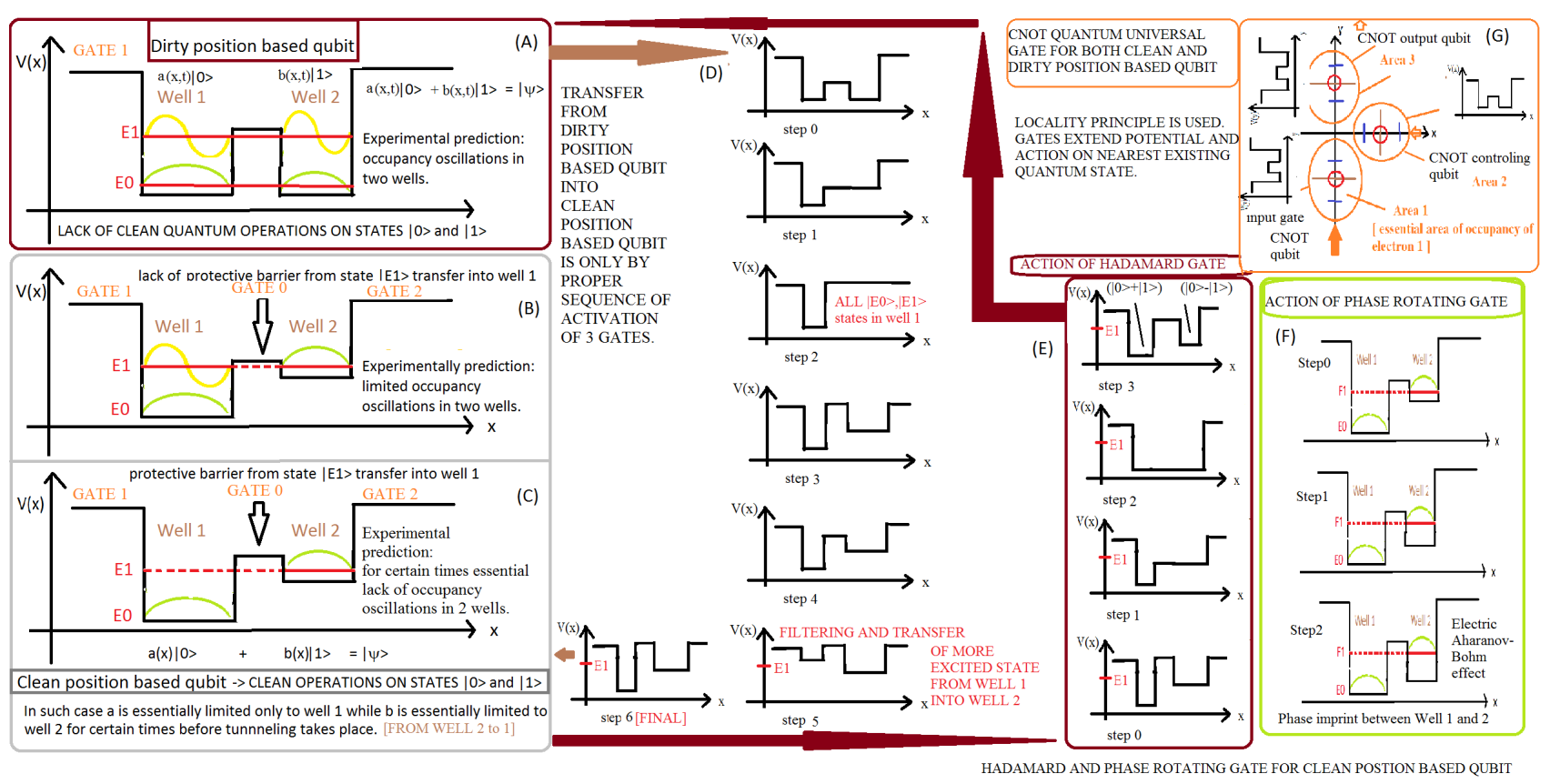

Figure 5. Concept of Wannier-based and eigenenergy-based electrostatic semiconductor qubit and universal gates built from eigenenergy-based position electrostatic superconducting qubits. The transfer from Wannier position based qubit (A) into eigenenergy-based position qubit (C) are shown by the sequence of steps illustrated by (D). The transfer from eigenenergy-based qubit to Wannier qubit are given by steps illustrated as (E). Action of phase rotating gate implemented in eigenenergy-based position qubit is given by $(\mathrm{F})$. Quantum CNOT gate common for both type of qubits is specified in Section (G).

\subsection{Interface between Semiconductor and Superconducting Qubit}

It is interesting to note that single-electron devices in case of superconducting technologies are manifested by superconducting Cooper pair box that uses concepts quite similar to that of the eigenenergy based semiconductor qubit described earlier in this work. External voltage can control the number of Cooper pairs in a small superconducting island and then can move them across the Josephson junction made by two superconducting areas separated by an insulator. The existing analogies are visualized in Fig.8. and in Fig.9. One can inspect the properties of semiconductor electrostatic qubit by monitoring the state of superconducting Cooper pair box or reversely. It seems that proper mathematical formalism for the study of both types of qubits is tightbinding formalism. Such a study can point the framework of hybrid semiconductor-superconducting computer architecture.

\section{MATHEMATICAL METHODOLOGY IN MODELING OF SEMICONDUCTOR QUANTUM CHIPS AND ITS COUPLING TO SUPERCONDUCTING QUBITS}

Quantum system dynamics with the lapse of time is described with the approach of Toeplitz matrix for the representation of kinetic energy term in Schrödinger equation.

In a $1 \mathrm{D}$ case with one particle, we have the following Hamiltonian

$$
H=-\frac{\hbar^{2}}{2 m_{1}} \frac{d^{2}}{d x_{1}^{2}}+V_{\mathrm{eff}}\left(x_{1}\right) .
$$

The action of this Hamiltonian $\hat{H} \psi(x)=E \psi(x)$ can be represented as $\psi\left(x_{s}\right)=\psi(s)$ since $x_{s}=x_{0}+s \Delta x$. 
Top view of CNOT

quantum gate

In nanostructures

CNOT input qubit

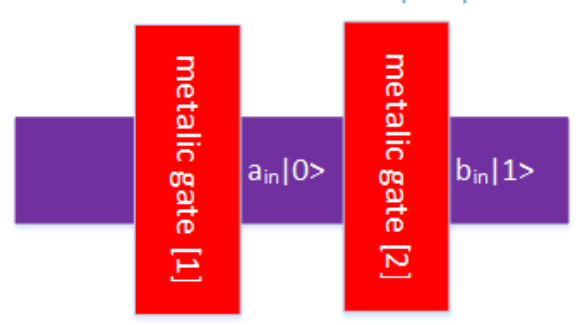

Output 2:determination of phase

difference between $\mathrm{a}_{\text {out, }}$ and $\mathrm{b}_{\text {out }}$

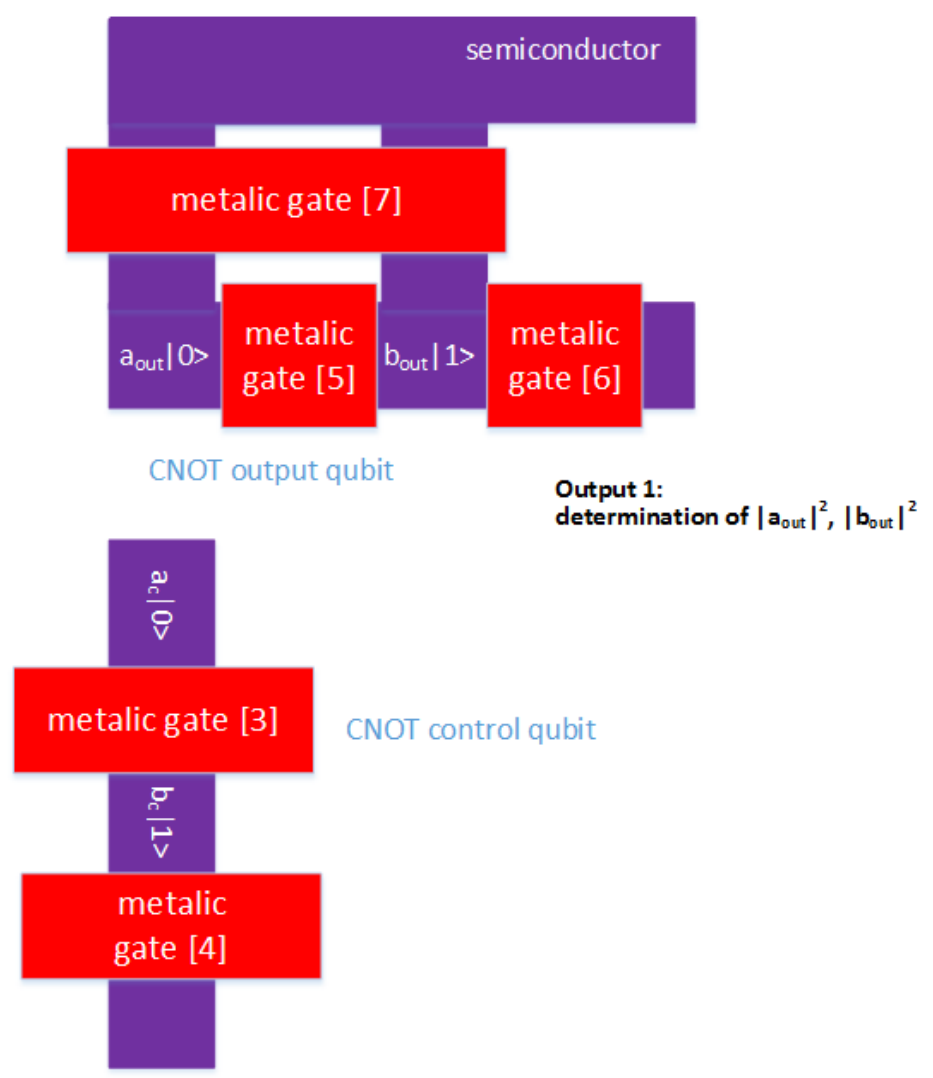

Figure 6. CNOT quantum gate based on position electrostatic qubits in both configurations of qubit.

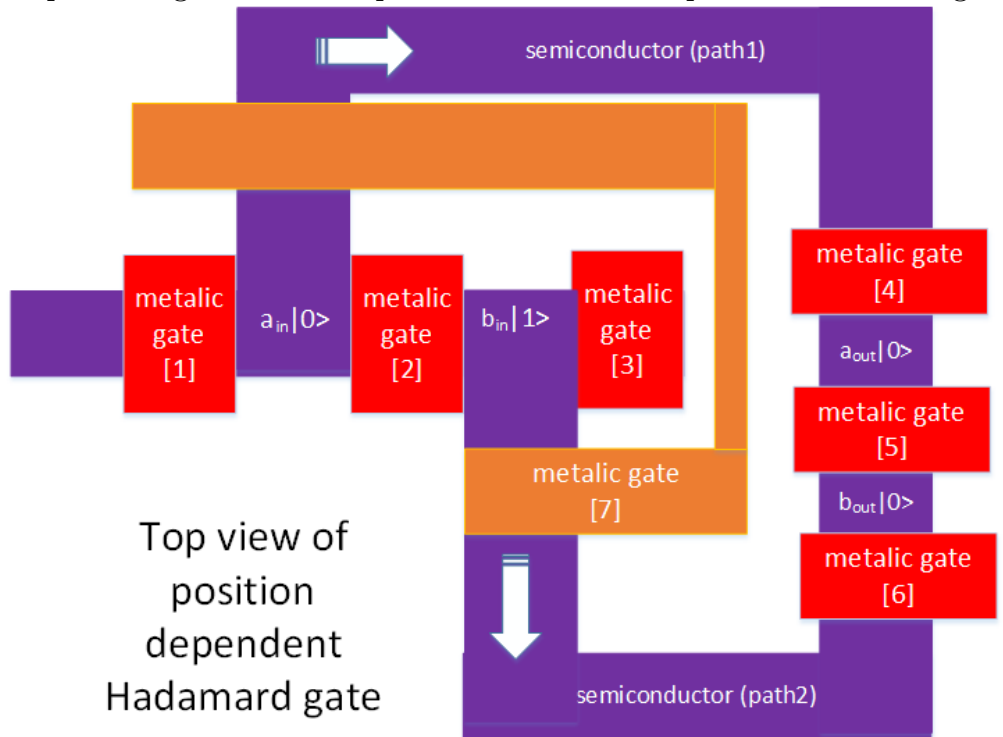

Figure 7. Hadamard quantum gate based on position electrostatic qubits in both configurations of qubit. 

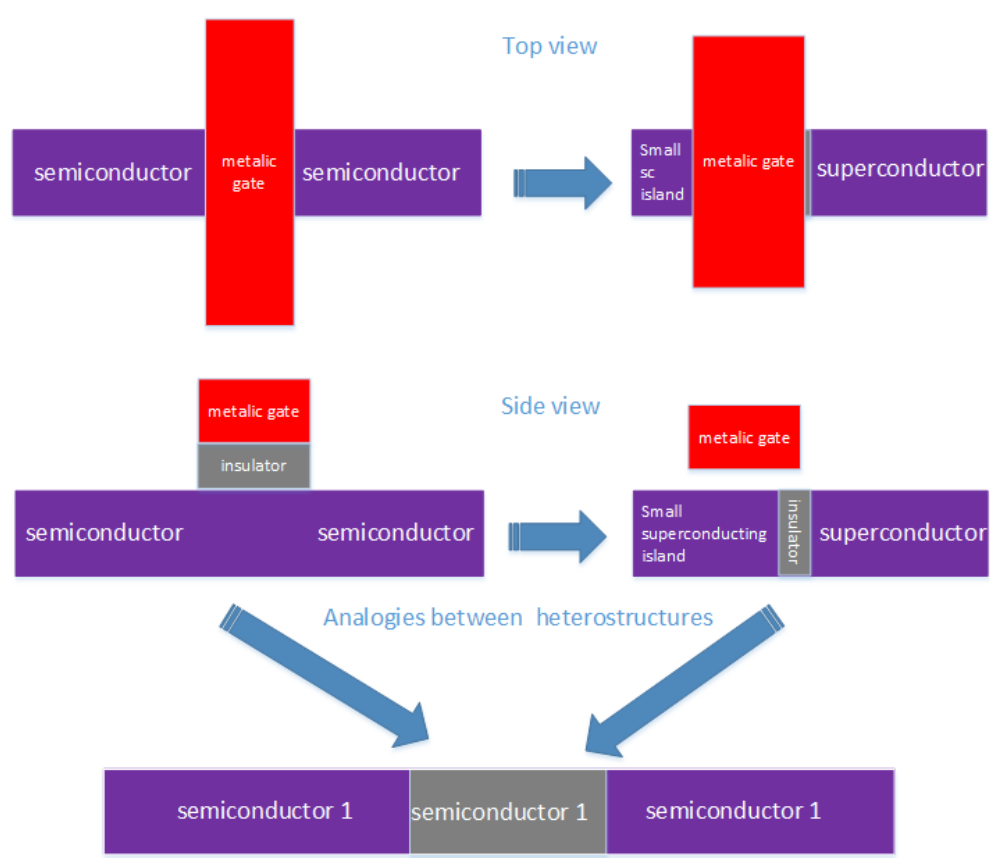

Figure 8. Comparison of semiconductor electrostatic qubits and superconductor Cooper pair box. Both devices are using single electron or single Cooper pair in potential landscape and are controlled electrostatically by external metalic gate. This analogies are helpful in construction the interface between semiconductor and superconducting qubits as it is depicted in Fig. 9.

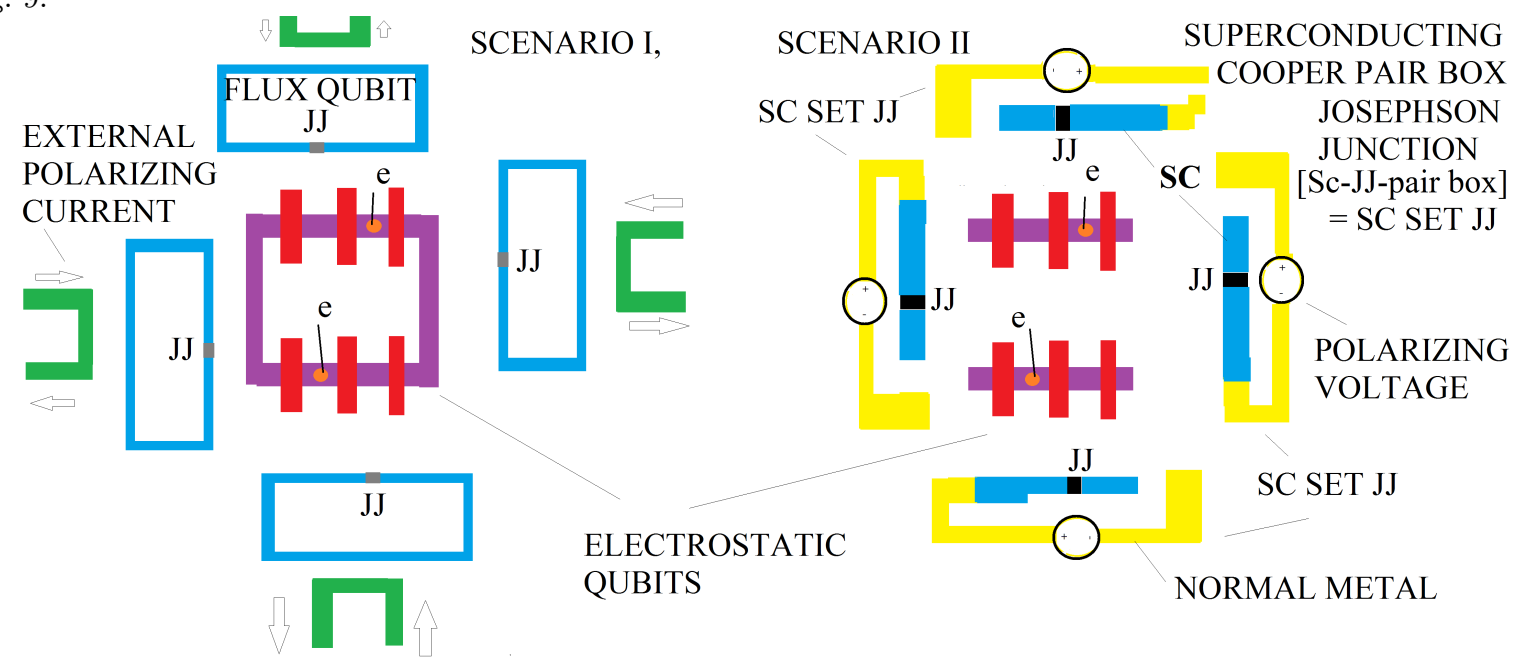

INTERFACE BETWEEN SUPERCONDUCTOR AND SEMICONDUCTOR QUBITS

Figure 9. Interface between position dependent electrostatic qubit and two types of superconducting qubit exploiting the similarity of controling mechanism of position dependent semiconductor qubits and Cooper pair box as depicted in Fig. 8 . Scenario I describes the interaction of flux superconducting qubits with electrostatic semiconductor qubits and Scenario II describes the interaction of superconducting Cooper pair boxes with electrostatic semiconductor qubits. 
The Hamiltonian can be written in a matrix representation as

$$
\frac{d^{2}}{d x^{2}}=\frac{1}{(\Delta x)^{2}}\left[\begin{array}{cccccccc}
-2 & 1 & 0 & 0 & 0 & 0 & 0 & 0 \\
1 & -2 & 1 & 0 & 0 & 0 & 0 & 0 \\
0 & 1 & -2 & 1 & 0 & 0 & 0 & 0 \\
\vdots & \ldots & \ldots & \ldots & \ldots & \ldots & \ldots & \vdots \\
0 & 0 & 0 & 0 & 1 & -2 & 1 & 0 \\
0 & 0 & 0 & 0 & 0 & 1 & -2 & 1 \\
0 & 0 & 0 & 0 & 0 & 0 & 1 & -2
\end{array}\right]
$$

In the most general case, the eigenstate of quantum system can be written in the following way at a given time instant $t$.

$$
\begin{array}{r}
|\psi(t)\rangle=q_{0}(t)|0(t)\rangle+q_{1}(t)|1(t)\rangle+\ldots+q_{n_{1}}(t)\left|n_{1}(t)\right\rangle+ \\
+\left(\int_{s 1(t)}^{s 2(t)} g\left(e_{1}, t\right) d e_{1}\right)\left|e_{1}(t)\right\rangle .
\end{array}
$$

The coefficients $q_{0}, . ., q_{n}$ fulfill the normalization condition $1=\left|q_{0}\right|^{2}+. .\left|q_{n}\right|^{2}+\int_{s 1(t)}^{s 2(t)}\left|g^{2}\right| d e_{1}$. The dynamics of quantum state gives

$$
i \hbar \frac{d}{d t}|\psi(t)\rangle=H(t)|\psi(t)\rangle=\hat{E}(t)|\psi(t)\rangle
$$

and can be written in discrete form as

$$
\begin{gathered}
|\psi(t+\Delta t)\rangle=|\psi(t)\rangle+\frac{-i \Delta t}{\hbar}(H(t+\Delta t)|\psi(t)\rangle) . \\
H(t)=E_{0}(t)|0(t)\rangle\left\langle 0(t)\left|+E_{1}(t)\right| 1(t)\right\rangle\langle 1(t)|+. .+ \\
+E_{n}(t)|n(t)\rangle\left\langle n(t)\left|+\int_{s 1(t)}^{s 2(t)} f\left(e_{1}, t\right) d e_{1}\right| e_{1}(t)\right\rangle\left\langle e_{1}(t)\right| \\
q_{0}(t+\Delta t)=\left(\langle 0(t+d t) \mid 0(t)\rangle q_{0}(t)+\langle 0(t+d t) \mid 1(t)\rangle q_{1}(t)+\ldots\right. \\
\left.+\langle 0(t+d t) \mid n(t)\rangle q_{n}(t)\right)+\ldots \\
q_{1}(t+\Delta t)=\left(\langle 1(t+d t) \mid 0(t)\rangle q_{0}(t)+\langle 1(t+d t) \mid 1(t)\rangle q_{1}(t)+\ldots\right. \\
\left.+\langle 1(t+d t) \mid n(t)\rangle q_{n}(t)\right)+\ldots \\
q_{n}(t+\Delta t)=\left(\langle n(t+d t) \mid 0(t)\rangle q_{0}(t)+\langle n(t+d t) \mid 1(t)\rangle q_{1}(t)+\ldots\right. \\
\left.+\langle n(t+d t) \mid n(t)\rangle q_{n}(t)\right)+\ldots
\end{gathered}
$$

In case of two interacting bodies confined by some local potentials, the Hamiltonian is as follows

$$
H=-\frac{\hbar^{2}}{2 m}\left(\left[\frac{d^{2}}{d x_{1}^{2}} \times I\right]+\left[I \times \frac{d^{2}}{d x_{2}^{2}}\right]\right)+\frac{e^{2}}{4 \pi \epsilon_{0}\left|x_{1}-x_{2}\right|}+V_{1}\left(x_{1}, t\right)+V_{2}\left(x_{2}, t\right)
$$

with $\psi\left(x_{1}, x_{2}\right)=\psi\left(x_{0}+i \Delta x, x_{0}+j \Delta x\right)=\psi_{i, j}$ and Euler scheme for 2 nd derivative. The initial state is $\left.\psi\left(x, y, t_{0}\right)=\sum_{i, j} q_{i, j} \psi_{i}\left(x_{1}, t_{0}\right) \psi_{j}\left(x_{2}, t_{0}\right)\right), \sum_{i, j}\left|q_{i, j}\right|^{2}=1$, which is a linear combination of non-interacting free particles with $E_{i}, E_{j}$ energies. 
The quantum state of electrostatically perturbatively interacting particles can be written by factorization of its state by formula

$$
\psi\left(x_{1}, x_{2}, t\right)_{p 1, p 2}=\psi\left(x_{1}, t\right)_{p 1} \psi\left(x_{2}, t\right)_{p 2} .
$$

Integrating the two-particle wavefunction either into $\psi\left(x_{1}, t\right)_{p 1}$ or into $\psi\left(x_{2}, t\right)_{p 2}$ gives us the following set of integro-differential equations:

$$
\begin{aligned}
& {\left[-\frac{\hbar^{2}}{2 m} \frac{d^{2}}{d x_{A}^{2}}+\lambda \frac{\int_{-\infty}^{+\infty} e^{2} \psi_{B}(x) \psi_{B}^{\dagger}(x) d x}{4 \pi \epsilon_{0}\left|x_{A}-x\right|}+V_{A}\left(x_{A}\right)\right] \psi_{A}\left(x_{A}\right)=E_{A} \psi_{A}\left(x_{A}\right)} \\
& {\left[-\frac{\hbar^{2}}{2 m} \frac{d^{2}}{d x_{B}^{2}}+\lambda \frac{\int_{-\infty}^{+\infty} e^{2} \psi_{A}(x) \psi_{A}^{\dagger}(x) d x}{4 \pi \epsilon_{0}\left|x_{B}-x\right|}+V_{B}\left(x_{B}\right)\right] \psi_{B}\left(x_{B}\right)=E_{B} \psi_{B}\left(x_{B}\right) .}
\end{aligned}
$$

The presented methodology is particularly effective in the mean field theory that has many manifestations in condensed matter physics. ${ }^{12}$ We have neglected the presence of spin in electron-electron interaction. Since electrons are fermions their wavefunction is antisymmetric with respect to electron change of position and spin at the same time. This brings formation of electron singlet or triplet state (or coexistence of both phases) in its dependence on electron wavefunctions overlap and distribution of external magnetic field forcing electron spin into certain dynamics. ${ }^{13}$ The existence of exchange interaction shall be treated in a rigorous way in order to account for spin effects that are usually much weaker than effects occurring from electrostatic interaction. Considerations on spin effects are beyond the scope of this work. The presented methodology is limited to 1 dimensional approach and therefore Ordinary Differential Equations (ODEs) are used. The detailed treatment of tackled problems in 2 and 3 space dimensions requires the use of Partial Differential Equations (PDEs). In certain conditions as in case of semiconductor or superconducting nanowires PDEs can be reduced to ODEs. Detailed study of PDEs requires the usage of Trotter matrices in 2 dimensions or requires the use of relaxation method as pointed in ${ }^{14,15}$ and is also beyond the scope of this work. Despite simplistic assumptions neglecting spin effects and treating system in one dimensional way the presented methodology can be used for quantum circuits and system of large integration under assumption of possible reduction from PDEs to ODEs.

Numerical and analytical solutions of the Schrödinger equation minimize the effective system Hamiltonian, which is especially visible when one uses functional derivatives ${ }^{15}$ that are the tools for derivation equations of motion from given functionals. In case of two electrostatically interacting particles confined by some local potential the expression

$$
\int_{-\infty}^{+\infty} \psi_{A}^{\dagger}\left(x_{A}\right) H_{B-e f f}\left[\psi_{A}, \psi_{B}\right] \psi_{B}\left(x_{A}\right) d x_{A}+\int_{-\infty}^{+\infty} \psi_{B}^{\dagger}\left(x_{B}\right) H_{B-e f f}\left[\psi_{A}, \psi_{B}\right] \psi_{B}\left(x_{B}\right) d x_{B}=\left\langle H_{\text {total }}\right\rangle
$$

is minimized with iterations, as shown in Eq. (34) and depicted in Fig. 11. The iterations allow to obtain the self consistency since the effective Hamiltonian depends on the state on which it acts.

Let us consider the Schrödinger equation with the discrete time step and a simplified Crank-Nicolson scheme. We have

$$
\hbar i \frac{\psi(x, t+\Delta t)-\psi(x, t)}{\Delta t}=\frac{1}{2}(H(t+d t) \psi(x, t+d t)+H(t) \psi(x, t)) .
$$

Alternatively, we can consider the extended Crank-Nicolsson scheme

$$
\begin{array}{r}
\hbar i \frac{\psi(x, t+\Delta t)-\psi(x, t)}{\Delta t}=\frac{1}{4}(H(t+d t) \psi(x, t+d t)+H(t) \psi(x, t)+H(t+d t) \psi(x, t)+H(t) \psi(x, d t+t))= \\
\frac{1}{4}(H(t+d t)+H(t))(\psi(t, x)+\psi(t+d t, x)) .
\end{array}
$$

In the $1 \mathrm{D}$ case, for $x=x_{0}+k \Delta x$ with $t=t_{0}+l d t \psi(x, t)=\psi(k)_{l}$ we have

$$
\frac{i \hbar}{\Delta t}\left(\psi(k)_{l+1}-\psi(k)_{l}\right)=\left(\frac{-\hbar^{2}}{4 m \Delta x^{2}}\left(\psi(k+1)_{l+1}-2 \psi(k)_{l+1}+\psi(k-1)_{l+1}\right)+\left(\psi(k+1)_{l}-2 \psi(k)_{l}+\psi(k-1)_{l}\right)\right) .
$$


We rewrite the equation as

$\frac{i \hbar}{\Delta t} \psi(k)_{l+1}+\frac{+\hbar^{2}}{4 m \Delta x^{2}}\left(\psi(k+1)_{l+1}-2 \psi(k)_{l+1}+\psi(k-1)_{l+1}\right)=\frac{-\hbar^{2}}{4 m \Delta x^{2}}\left(\psi(k+1)_{l}-2 \psi(k)_{l}+\psi(k-1)_{l}\right)-\frac{i \hbar}{\Delta t} \psi(k)_{l}$.

Setting coefficients $\alpha=\frac{i \hbar}{\Delta t}$ and $\beta=\frac{+\hbar^{2}}{4 m \Delta x^{2}}$ we obtain

$(\alpha-2 \beta)\left(\psi(k)_{l+1}\right)+\beta\left(\psi(k+1)_{l+1}+\psi(k-1)_{l+1}\right)=-\beta\left(\psi(k+1)_{l}+\psi(k-1)_{l}\right)-(\alpha+2 \beta)\left(\psi(k)_{l}\right)=\gamma(k-1, k, k+1)_{l}$.

The right-hand side is dependent on $l$ th time step and left side is dependent on $(l+1)$ th time step. We express the previous equation in a matrix form as

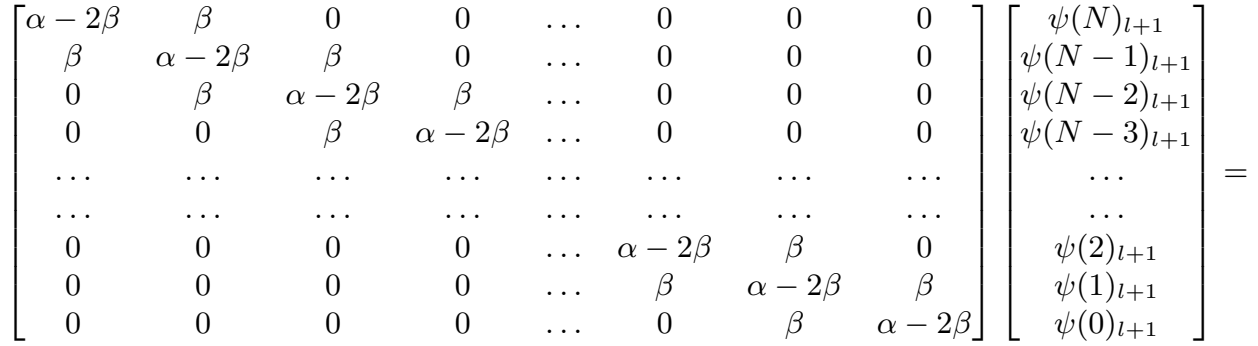

$$
\begin{aligned}
& -(\alpha+2 \beta)\left[\begin{array}{c}
\psi(N)_{l} \\
\psi(N-1)_{l} \\
\psi(N-2)_{l} \\
\psi(N-3)_{l} \\
\cdots \\
\cdots \\
\psi(2)_{l} \\
\psi(1)_{l} \\
\psi(0)_{l}
\end{array}\right]-\beta\left[\begin{array}{c}
\psi(N+1)_{l}+\psi(N-1)_{l} \\
\psi(N)_{l}+\psi(N-2)_{l} \\
\psi(N-1)_{l}+\psi(N-3)_{l} \\
\psi(N-2)_{l}+\psi(N-4)_{l} \\
\cdots \\
\cdots \\
\psi(3)_{l}+\psi(1)_{l} \\
\psi(2)_{l}+\psi(0)_{l} \\
\psi(1)_{l}+\psi(-1)_{l}
\end{array}\right]=-(\alpha+2 \beta)\left[\begin{array}{c}
\psi(N)_{l} \\
\psi(N-1)_{l} \\
\psi(N-2)_{l} \\
\psi(N-3)_{l} \\
\cdots \\
\ldots \\
\psi(2)_{l} \\
\psi(1)_{l} \\
\psi(0)_{l}
\end{array}\right]-\beta\left[\begin{array}{c}
\psi(N-1)_{l} \\
\psi(N)_{l}+\psi(N-2)_{l} \\
\psi(N-1)_{l}+\psi(N-3)_{l} \\
\psi(N-2)_{l}+\psi(N-4)_{l} \\
\ldots \\
\ldots \\
\psi(3)_{l}+\psi(1)_{l} \\
\psi(2)_{l}+\psi(0)_{l} \\
\psi(1)_{l}
\end{array}\right] .
\end{aligned}
$$

The tridiagonal matrix can be inverted $(\operatorname{inv}[\hat{M}]$ states for inverted matrix $\hat{M})$ and we obtain the dependence for the $(l+1)$ th time step from the knowledge of wavefunction at the $l$ th time step. We have

$$
\begin{aligned}
& \left.\begin{array}{c}
\psi(N)_{l+1} \\
\psi(N-1)_{l+1} \\
\psi(N-2)_{l+1} \\
\psi(N-3)_{l+1} \\
\cdots \\
\cdots \\
\psi(2)_{l+1} \\
\psi(1)_{l+1} \\
\psi(0)_{l+1}
\end{array}\right]=
\end{aligned}
$$

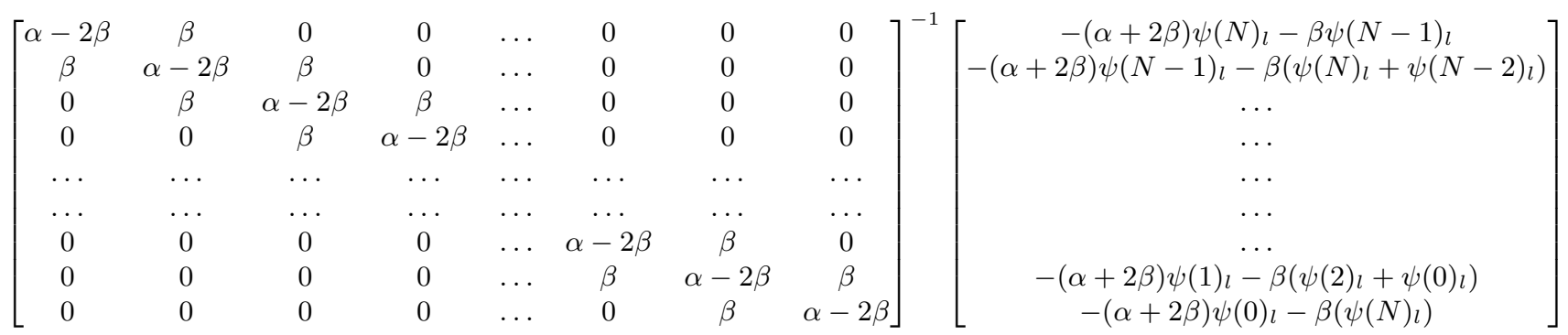

It is thus shown that the time-dependent dynamics of quantum state in one dimension can be treated by the matrix approach. The accuracy of this numerical approach is dependent on the discretization of space $d x$ and discretization of time by $d t$ time step. 


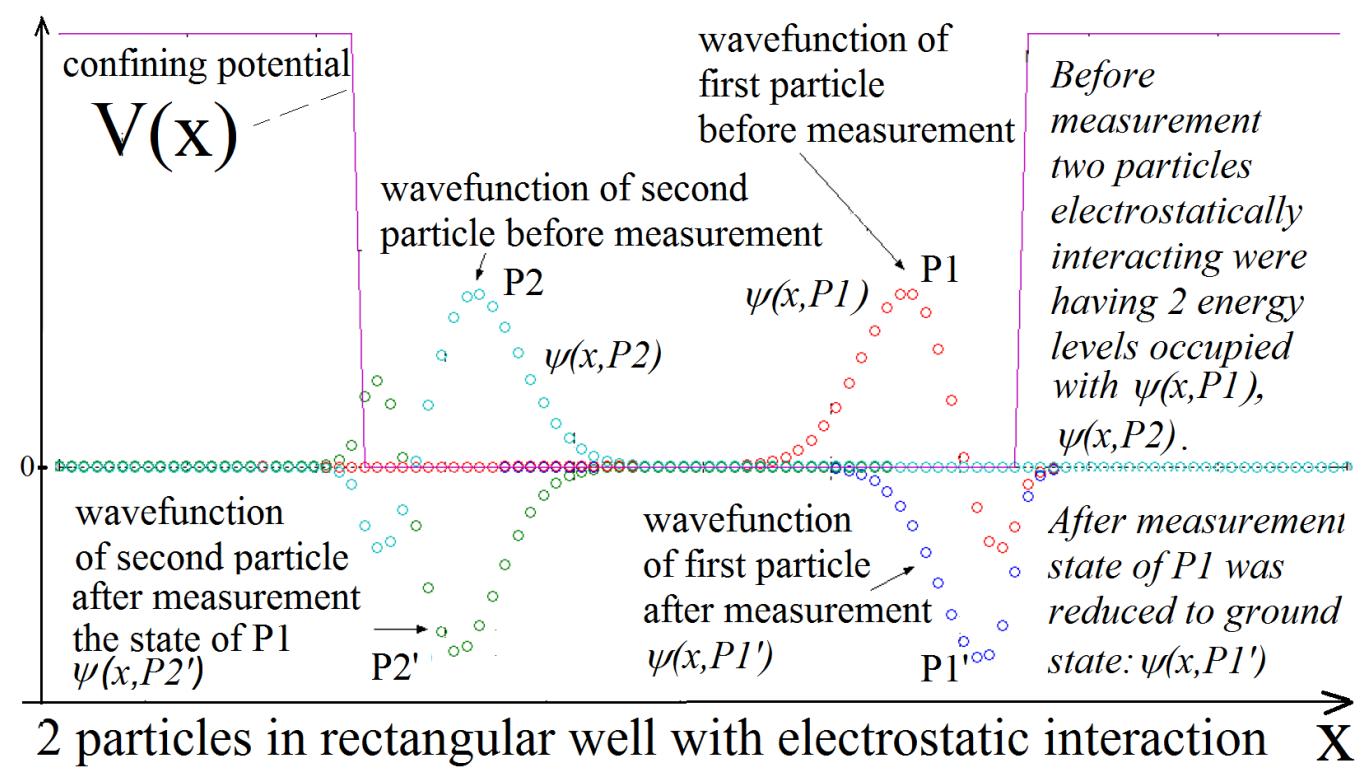

Figure 10. Wavefunctions of 2 electrostatically interacting particles with 2 energetic levels confined in rectangular potential before and after measurement of the quantum state of one particles that brings the collapse of one particle wavefunction into one energy level of measured particle.

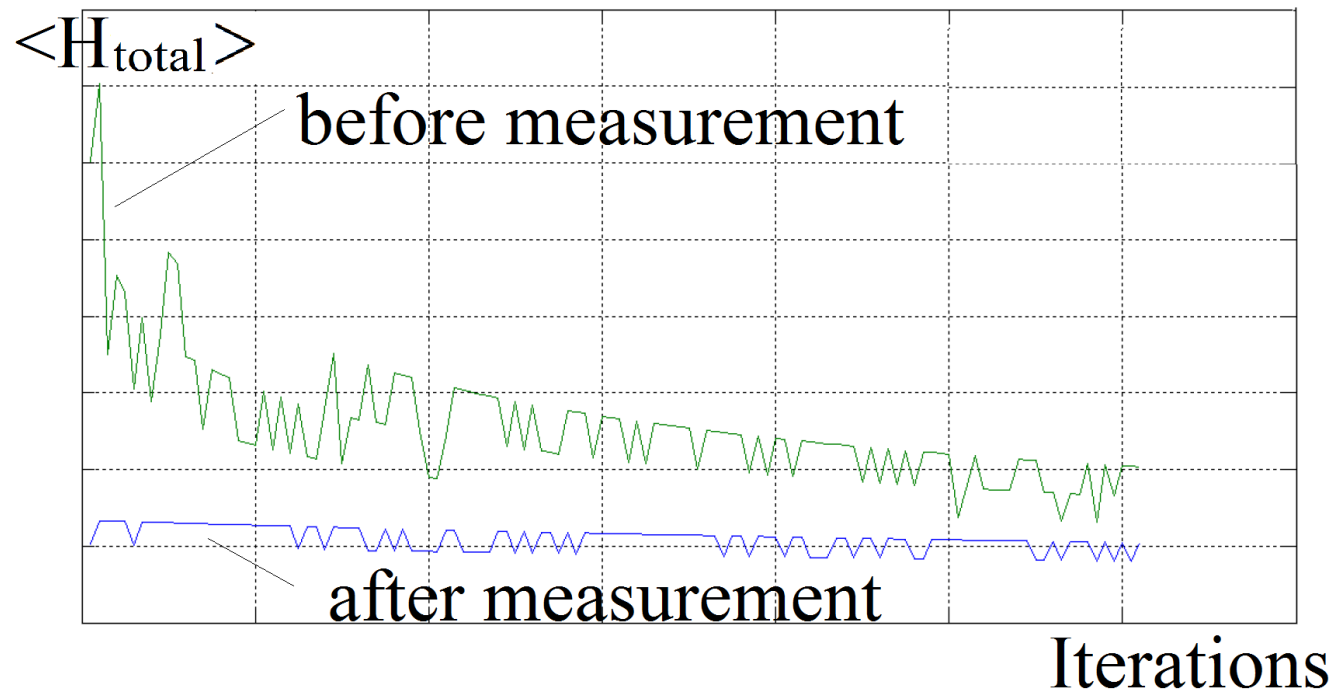

Figure 11. Effective system Hamiltonian for 2 electrostatically coupled electrons given by equation 34 and Fig. 10 . with next iterations for the physical situation of lack of measurement and with strong measurement being projection on one electron energy base. 


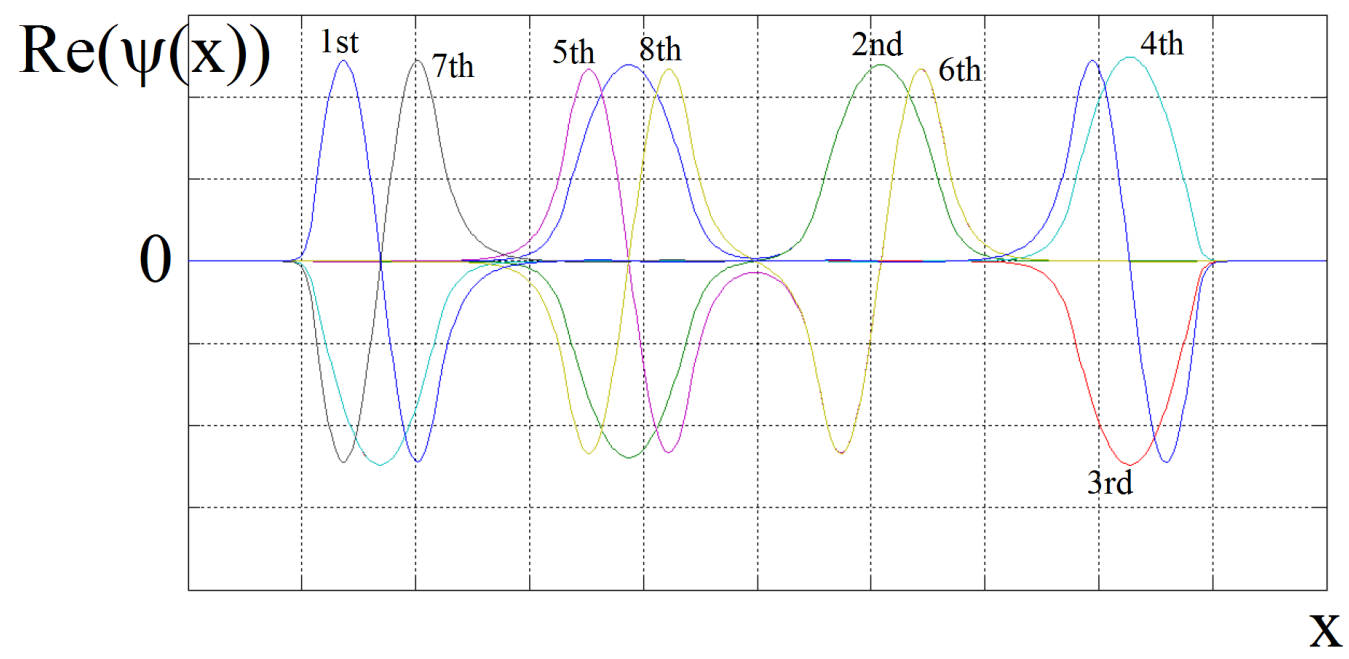

Figure 12. Lowest energy eigenfunctions in the system of 8 coupled quantum wells (as generalizatio of the system from upper part of Fig. 1.) obtained by Toeplitz matrix approach.

\section{PRELIMINARY NUMERICAL RESULTS ON POSITION-DEPENDENT ELECTROSTATIC QUBIT, PHASE ROTATING QUANTUM GATE, HADAMARD GATE AND CNOT GATE}

At first, we test the numerical algorithm using Toeplitz matrices in case of Hamiltonian that is time independent. Wavefunctions of two electrostatically interacting particles with two energy levels confined in a rectangular potential are obtained before and after the measurement of quantum state of one of the particles that brings the collapse of wavefunction into one energy level of measured particle. It is depicted in Fig. 10. During the execution of this algorithm, certain iterations are performed so that self-consistency of the distribution of two wavefunctions being codependent are obtained. The effective system Hamiltonian with iterations is depicted in Fig. 11. The algorithm was also tested for the case of chain of eight coupled quantum dots so we have eight wells in the effective potential. The example of obtained eigenstates is depicted in Fig. 12.

The next step in the numerical modeling of quantum gates for both types of position-dependent electrostatic semiconductor qubits is the case of phase-rotating gate that is the simplest one in this approach. Certain idea behind the operation of phase-rotating gate is by the use of magnetic or electric Aharanov-Bohm effect. The magnetic Aharonov-Bohm effect is visualized in Fig. 1.2 and its electric version is visualized in Fig.4. We are using Crank-Nicolson algorithm that is a well-known method for solving the diffusion equation with a Toeplitz matrix approach. In order to simulate its operation principle, we place the particle in two rectangular potentials so we can have the system of two partly connected wells separated by some middle barrier. Then we start to gradually increase the medium barrier and start to increase the right well bottom, as well as rightmost barrier, as indicated in Fig. 14. In such a way, we can nearly preserve the occupancy in each well. During the phase imprinting, the occupancy is only slightly changed, as demonstrated in Fig. 15, while the phase imprint was generated in Fig. 13. The obtained results show that we can move around the Bloch sphere changing one of angles that parameterize the quantum state. However, a change in one of the angles brings a certain change to another angle. This shows that we can have certain controlability on quantum state but we can only achieve certain precision in setting up the quantum state. It should be of no surprise that in the technological realization the phase rotating gate is non-ideal as it is the case of any circuit element. What is more inevitably by the act of changing the effective potential is that we can only generate a certain limited number of excited states. Those excited states can be later de-excited with some additional action of CMOS voltage gates. However it is beyond the scope of this work.

During this operation we use the principle of electric Aharonov-Bohm effect. The alternative and more desirable phase control across the semiconductor position-dependent qubits is by the use of magnetic AharonovBohm effect that is visualized in Fig. 1.2. This effect is commonly used in superconducting electronics and 
bringing up a smaller disturbance to the quantum state that is being phase imprint than is the case of the electric Aharonov-Bohm effect. Thus, the magnetic control of phase difference could appear preferable from the qubit design point of view. However, it is more costly technologically since it requires extra external circuitry generating the vector potential.

The next type of universal quantum gate in our treatement is Hadamard gate (HG). For a given qubit input it assigns a qubit output what schematically can be written as

$$
H G(\mid i>)=\left\{\begin{array}{l}
|i>=| 0\rangle \rightarrow \frac{1}{\sqrt{2}}(|0\rangle+|1\rangle) \\
|i>=| 1\rangle \rightarrow \frac{1}{\sqrt{2}}(|0\rangle-|1\rangle)
\end{array} .\right.
$$

In the case of Wannier position-dependent electrostatic qubit, we should expect that, after this operation, the neighboring wells will be 'half-way' occupied. Indeed, this is possible if we consider the situation as depicted in Fig. 16. The wavefunction in two interconnected wells with no-separating barrier and the same well bottoms is being 'cut' by the steady increase of the middle barrier that separates the two wells. The circuit implementing the action of Hadamard gate is depicted in Fig. 7. The presented numerical results refer to the action of the middle barrier. Once the final state is obtained, it can be transported to some other part of the quantum circuit. This can be achieved by turning off the confinement potential $V_{3}$. Then, the wavepackets will go along different paths what will cause their different phase evolution. Once they arrive to the right-most side of the structure, the confinement potential $V_{1 Q}$ and $V_{2 Q}$ can be turned on and the quantum state will be localized in the right part of circuit. Depending on the desired Hadamard gate output, this state can be proportional to $|0\rangle+|1\rangle$ and to $|0\rangle-|1\rangle$. The positive or negative value of the sign can be regulated by adding additional AC bias signal to the $V_{3}$ potential. More detailed Hadamard gate operation would be a subject of a future study.

Modeling time dependent dynamics of two weakly electrostatically interacting electrons can be achieved with the use of Toepltiz matrix approach with Cranck-Nicolson scheme that can represent time-dependent integrodifferential equations. We consider the system when two electrons have a superposition of two energy levels. If they would be in parallel quantum wires, they tend to minimize their electrostatic energy. It is visible in they phase anticorrelation as depicted in Fig. 17. Phase anticorrelation simply means that electrons move in opposite directions. However if two electrons are in the same nanowire one can obtain their phase correlation and synchronization of their movement. In such way the system electrostatic energy can be minimized.

In order to model CNOT gate as depicted in Fig. 1 and in Fig. 6. one needs to solve the problem of 3 interacting electrons confined by some local potentials in 3 different nanowires. The quantum state of one electron is used as input CNOT qubit, while the state of neighbouring electron in neighbouring quantum wire is used as the controlling qubit. The quantum state of third electron is used as CNOT output. The essential concept that behind the CNOT gate operation is the weak Coulomb electron-electron interaction (inductive coupling). Thus it is possible to describe the action of CNOT gate by the set of 3 coupled integro-differential equations. In the considered 3-body case the electron degrees of freedom are limited by the presence of local confing potentials. The full treatment of CNOT gate is by rigorous solutions of 3-body problem that is beyond the scope of this work but is in the capabilities of described numerical method using Toeplitz matrices readapted for 2 and 3-body case.

Numerical results obtained from described numerical methods show that it is hard to obtain full reversibility of quantum gate actions. Also we are dealing with the situation when quantum state is being heated during the action of universal gates. Preliminary results show that it is necessary to use additional signal patterns applied to CMOS polarizing gates that will bring deexcitment to the obtained quantum state. After all the prize to be paid for very quick quantum gate operation is the increase of system entropy that brings dissipative processes and thus makes technologically implemented quantum gates not to be fully reversible.

Using the given reasoning we can design wide class of quantum universal gates that is : swap gate, Toeplitz quantum gate, Toffoli gate. This is however beyond the scope of this work. It is noticable to underline that physical structure supporting proper quantum gate action is the same for both type of position dependent qubits. 


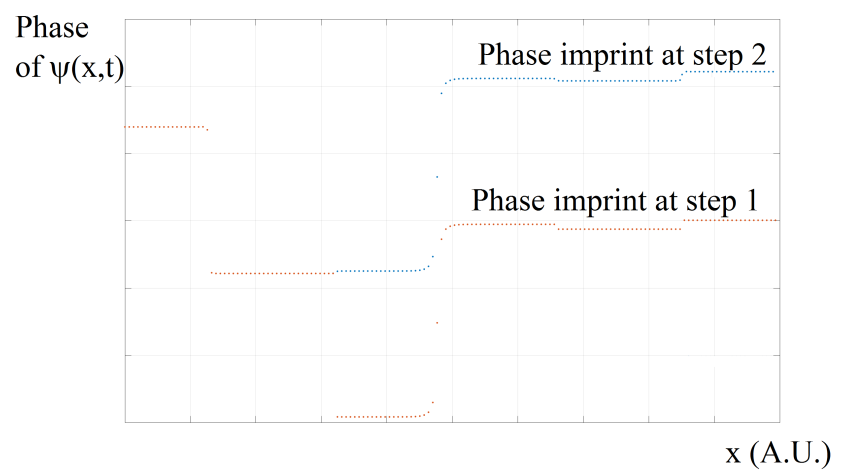

Figure 13. Distribution of wavefunction phase in space in Wannier qubit after action of phase rotating gate. The electrostatic Aharonov-Bohm effect is exploited due to movement of wells bottoms as given by Fig. 14. We shall underline that Phase Rotating Gate is also necessary for the proper operation of Hadamard gate.

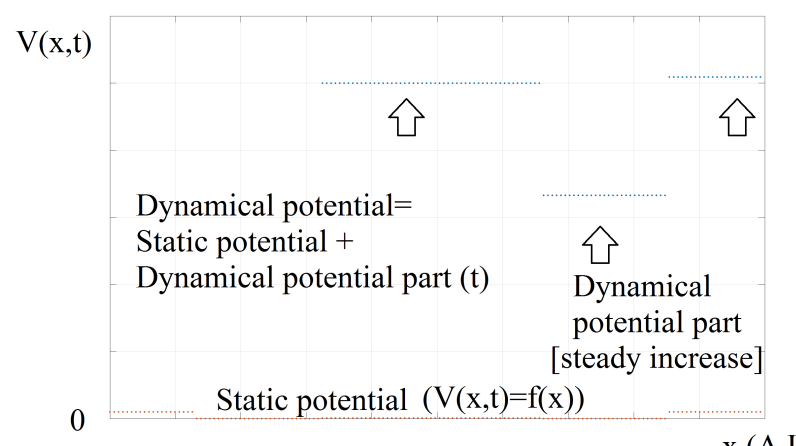

x (A.U.)

Figure 14. Steady potential change with time as a function of position in phase rotating gate that generates phase distribution as given in Fig. 13.

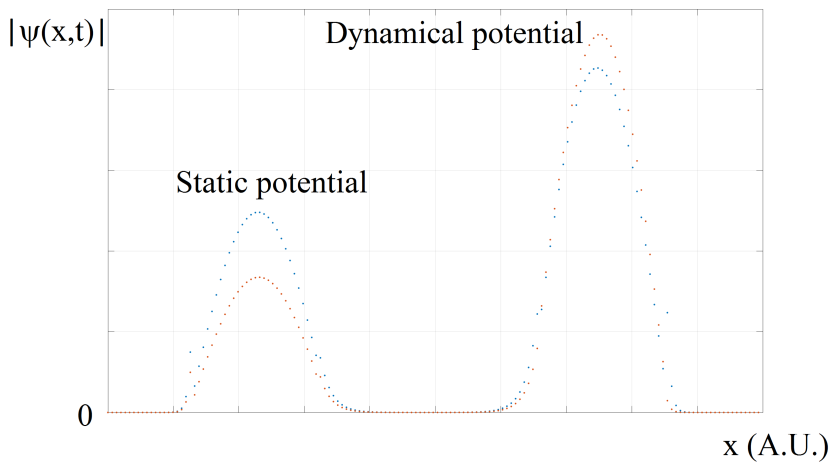

Figure 15. Real-valued parts of wavefunction in function of position in phase rotating gate before and after phase imprinting due to effective potential change with time as given by Fig. 14.

What is more we can achieve the reverse action of Hadamard gate when we transfer the quantum state from eigenenergy-based position depedent qubit (Fig. 5 C) to Wannier-based position dependent qubit as it is given by Fig. $5 \mathrm{~A}$ going around path 5 E. The action of reverse Hadmard gate is also achieved when we transform Wannier-based qubit to eigenenergy-based qubit what is given by path from Fig. 5 D. It is remarkable that realizing electrical phase control on the both type of qubits we are dealing with situation from Fig. 5 F. CNOT gate for both type of qubits is given by Fig. $5 \mathrm{G}$ and by Fig. 6 . 

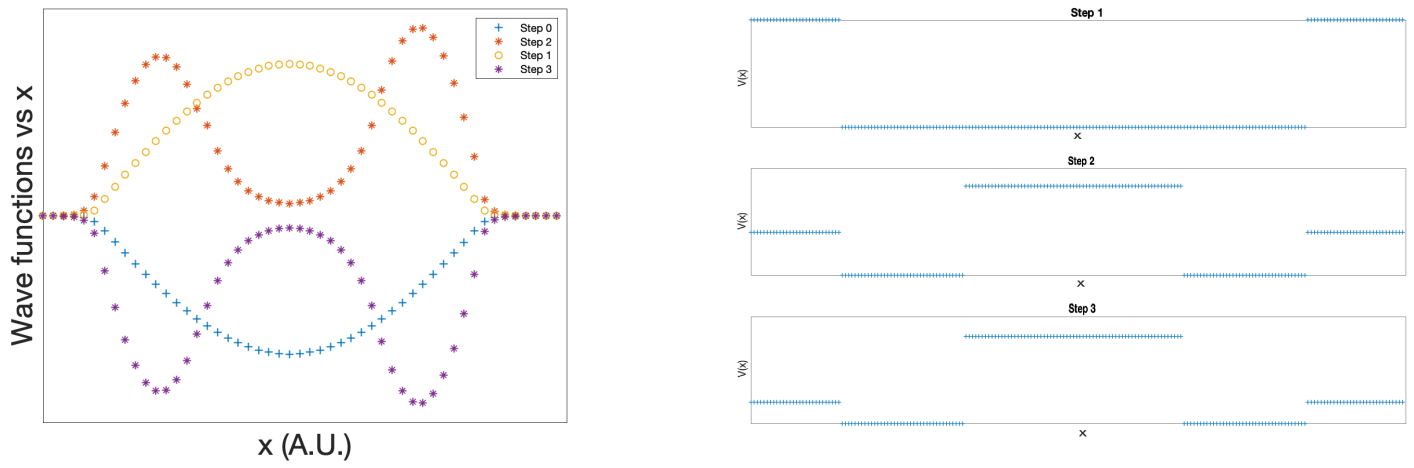

Figure 16. Change of wavefunction during action of potential separating one rectangular well into two rectangular wells. The final state of wavefunction mimics the action of Hadamard gate that transfers $|0\rangle$ into $\frac{1}{\sqrt{2}}(|0\rangle+|1\rangle)$. Additional phase imprinting across the system is possible in order to mimic the transfer from $|1\rangle$ into $\frac{1}{\sqrt{2}}(|0\rangle-|1\rangle)$. The philosophy behind phase control across 2 wells was already included in the principle of operation of phase rotating gate that is illustrated by Fig. 14, 13, 15 .

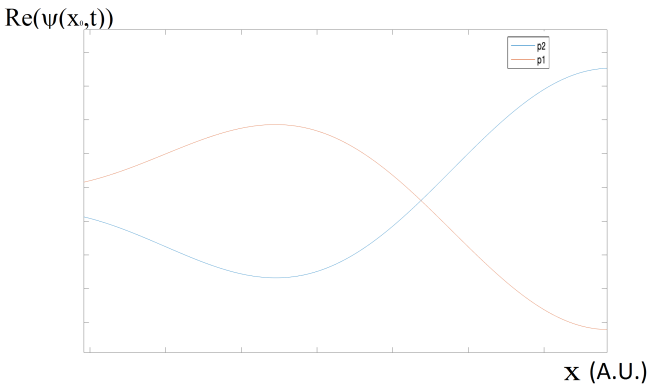

Figure 17. Phase anticorrelation occurring in 2 perturbatively electrostatically interacting electrons $p_{1}$ and $p_{2}$ placed in different confining potentials that are in parallel one to each other as it is given by the middle section of Fig. 9.Phase anticorrelation shows that electrons move in opposite directions trying to minimize their electrostatic energy what is used in the design of CNOT gate whose structure is depicted in Fig. 6.

\section{CONCLUSIONS}

Basic methodology in modeling of semiconductor electrostatic qubit and quantum universal gates was used and preliminary numerical results were obtained. The forthcoming issues are as follows:

- Non-equilibrium dynamics included in modeling of quantum gates

- Modeling of classical CMOS at low temperatures

- Extension of numerical scheme to coupled non-linear integro partial differential equations between semiconductor qubit (Schroedinger integro-differential equations ) and integro-differential Bogoliubov-de Gennes ${ }^{14}$ equations (superconducting qubit)

- Probing the dynamics of coupled position dependent electrostatic qubits and quantum universal gates by its interaction with superconducting qubits as given by Fig. 9 .

Various experiments using the derived concepts expressed in certain structure of physical systems can be conducted. The applied numerical methods need to be extended to cover the cases of circuit with complex topology. It can be achieved by the usage of relaxation method. ${ }^{15}$ Also for the case of massive quantum circuits with CMOS qubits and quantum gates it is instructive to conduct the canonical quantization of classical equations of motion. 
Once we consider the interface between semiconductor-superconductor quantum computer we shall exercise the procedure presented in. ${ }^{16}$ The remarkable future of both semiconductor and superconducting technologies is the ability to built field induced Single Electron Devices. The field used in semiconductor is the electric while the field used to control transport properties in superconductor is magnetic. It is thus recommendable to trace methodological steps presented in. ${ }^{14}$ The mentioned issues are the subject of future work.

\section{ACKNOWLEDGMENT}

We would like to thank to Adam Bednorz from University of Warsaw and to Amir Bozorg from University College Dublin for helpful discussions and comments. This work was supported by Science Foundation Ireland under Grant 14/RP/I2921.

\section{REFERENCES}

1. T. T. D. Ladd et al, "Quantum computers," Nature 464, pp. 45-53, 2010.

2. C. Lai et al., "Coherent zero-state and p-state in an exciton-polariton condensate array," Nature 450, pp. 529-532, 2007.

3. 'T.Hey, "Quantum computing: an introduction," Computing and Control Engineering Journal 10, pp. 105$112,1999$.

4. E. Rieffel and W. Polak, "An introduction to quantum computing for non-physicists," ACM Computing Surveys (CSUR) 32(3), pp. 300-335, 2000.

5. N. M. Linke, D. Maslov, M. Roetteler, S. Debnath, C. Figgatt, K. A. Landsman, K. Wright, and C. Monroe, "Experimental comparison of two quantum computing architectures," Proceedings of the National Academy of Sciences 114(13), pp. 3305-3310, 2017.

6. J. You and F. Nori, "Atomic physics and quantum optics using superconducting circuits," Nature 474(589), 2011.

7. D. Leipold, "Controlled Rabi Oscillations as foundation for entangled quantum aperture logic," in Seminar at Berkley Lab, 25th July 2018.

8. P. Giounanlis, E. Blokhina, D. Leipold, and R. B. Staszewski, "Occupancy oscillations and electron transfer in multiple-quantum-dot qubits and their circuit representation," 25th IEEE International Conf. on Electronics Circuits and Systems (ICECS), pp. 153-156, 2018.

9. G. Shinkai, T. Hayashi, T. Ota, and T. Fujisawa, "Correlated coherent oscillations in coupled semiconductor charge qubits," Phys. Rev. Lett. 103, p. 056802, Jul 2009.

10. K. D. Petersson, J. R. Petta, H. Lu, and A. C. Gossard, "Quantum coherence in a one-electron semiconductor charge qubit," Phys. Rev. Lett. 105, p. 246804, Dec 2010.

11. P. Giounanlis, E. Blokhina, K. Pomorski, D. Leipold, and R. B. Staszewski, "Semiconductor electrostatic qubits realized through coupled quantum dots, in review," in review, 2019.

12. J. C. Slater, "A simplification of the hartree-fock method," Phys. Rev. 81, pp. 385-390, 1951.

13. J. Spalek, "Wstep do fizyki materii skondensowanej," $P W N, 2015$.

14. K.Pomorski and P.Prokopow', "Possible existence of field induced josephson junction," Physica Status Solidi B 249(9), pp. 1805-1813, 2012.

15. K. D. Pomorski, H. Akaike, A. Fujimaki, and K. Rusek, "Relaxation method in description of ram memory cell in rsfq computer," COMPEL - The international journal for computation and mathematics in electrical and electronic engineering $\mathbf{0}(0)$, p. null, 0.

16. K. Pomorski and A. Bednorz, "Justification of the canonical quantization of the josephson effect and its modifications due to high capacitance energy," Journal of Physics A: Mathematical and Theoretical 49(12), p. $125002,2016$. 